\title{
Correlation Functions for a Chain of Short Range Oscillators
}

\author{
T. Grava ${ }^{1,2} \cdot$ T. Kriecherbauer $^{3}$ (1) - G. Mazzuca ${ }^{1}$ (1) $\cdot$ K. D. T.-R. McLaughlin ${ }^{4}$
}

Received: 14 October 2020 / Accepted: 17 February 2021 / Published online: 18 March 2021

(c) The Author(s) 2021

\begin{abstract}
We consider a system of harmonic oscillators with short range interactions and we study their correlation functions when the initial data is sampled with respect to the Gibbs measure. Such correlation functions display rapid oscillations that travel through the chain. We show that the correlation functions always have two fastest peaks which move in opposite directions and decay at rate $t^{-\frac{1}{3}}$ for position and momentum correlations and as $t^{-\frac{2}{3}}$ for energy correlations. The shape of these peaks is asymptotically described by the Airy function. Furthermore, the correlation functions have some non generic peaks with lower decay rates. In particular, there are peaks which decay at rate $t^{-\frac{1}{4}}$ for position and momentum correlators and with rate $t^{-\frac{1}{2}}$ for energy correlators. The shape of these peaks is described by the Pearcey integral. Crucial for our analysis is an appropriate generalisation of spacings, i.e. differences of the positions of neighbouring particles, that are used as spatial variables in the case of nearest neighbour interactions. Using the theory of circulant matrices we are able to introduce a quantity that retains both localisation and analytic viability. This also allows us to define and analyse some additional quantities used for nearest neighbour chains. Finally, we study numerically the evolution of the correlation functions after adding nonlinear perturbations to our model. Within the time range of our numerical simulations the asymptotic description of the linear case seems to persist for small nonlinear perturbations while stronger nonlinearities change shape and decay rates of the peaks significantly.
\end{abstract}

Communicated by Herbert Spohn.

$\triangle$ T. Kriecherbauer

thomas.kriecherbauer@uni-bayreuth.de

T. Grava

grava@sissa.it

G. Mazzuca

guido.mazzuca@sissa.it

K. D. T.-R. McLaughlin

kenmcl@rams.colostate.edu

1 SISSA, via Bonomea 265, 34136 Trieste, Italy

2 School of Mathematics, University of Bristol, Fry Building Woodland Road, Bristol BS8 1UG, UK

3 Department of Mathematics, Universitat Bayreuth, Bayreuth, Germany

4 Department of Mathematics, Colorado State University, 1874 Campus Delivery, Fort Collins, CO 80523, USA 
Keywords Harmonic chains - Anharmonic chains - Short range interactions · Equilibrium time correlations $\cdot$ Airy scaling $\cdot$ Pearcey scaling

\section{Introduction}

In this manuscript we consider a system of $N=2 M+1$ particles interacting with a short range harmonic potential with Hamiltonian of the form

$$
H=\sum_{j=0}^{N-1} \frac{p_{j}^{2}}{2}+\sum_{s=1}^{m} \frac{\kappa_{s}}{2} \sum_{j=0}^{N-1}\left(q_{j}-q_{j+s}\right)^{2},
$$

where $1 \leq m \ll N, \kappa_{1}>0, \kappa_{m}>0$, and $\kappa_{s} \geq 0$ for $1<s<m$. In order to make sense of (1.1) we need to introduce boundary conditions. Throughout this paper we consider periodic boundary conditions. By that we mean that the indices $j$ are taken from $\mathbb{Z} / N \mathbb{Z}$ and therefore

$$
q_{N+j}=q_{j}, \quad p_{N+j}=p_{j}
$$

holds for all $j$. The Hamiltonian (1.1) can be rewritten in the form

$$
H(\mathbf{p}, \mathbf{q}):=\frac{1}{2}\langle\mathbf{p}, \mathbf{p}\rangle+\frac{1}{2}\langle\mathbf{q}, A \mathbf{q}\rangle,
$$

where $\mathbf{p}=\left(p_{0}, \ldots, p_{N-1}\right), \mathbf{q}=\left(q_{0}, \ldots, q_{N-1}\right),\langle.,$.$\rangle denotes the standard scalar product$ in $\mathbb{R}^{N}$ and where $A \in \operatorname{Mat}(N, \mathbb{R})$ is a positive semidefinite symmetric circulant matrix generated by the vector $\mathbf{a}=\left(a_{0}, \ldots, a_{N-1}\right)$ namely $A_{k j}=a_{(j-k) \bmod N}$ or

$$
A=\left[\begin{array}{ccccc}
a_{0} & a_{1} & \ldots & a_{N-2} & a_{N-1} \\
a_{N-1} & a_{0} & a_{1} & & a_{N-2} \\
\vdots & a_{N-1} & a_{0} & \ddots & \vdots \\
a_{2} & & \ddots & \ddots & a_{1} \\
a_{1} & a_{2} & \ldots & a_{N-1} & a_{0}
\end{array}\right],
$$

where

$$
a_{0}=2 \sum_{s=1}^{m} \kappa_{s}, \quad a_{s}=a_{N-s}=-\kappa_{s}, \quad \text { for } s=1, \ldots, m \text { and } a_{s}=0 \text { otherwise. }
$$

Due to the condition $\kappa_{1}>0$ we have $\langle\mathbf{q}, A \mathbf{q}\rangle=0$ iff all spacings $q_{j+1}-q_{j}$ vanish. Therefore the kernel of $A$ is one-dimensional with the constant vector $(1, \ldots, 1)^{\top}$ providing a basis. This also implies that the lattice at rest has zero spacings everywhere. Observe, however, that one may introduce an arbitrary spacing $\Delta$ for the lattice at rest by the canonical transformation $Q_{j}=q_{j}+j \Delta, P_{j}=p_{j}$ which does not change the dynamics. The periodicity condition for the positions $Q_{j}$ then reads $Q_{N+j}=Q_{j}+L$ with $L=N \Delta$ (see e.g.[17, Sect. 2]).

The harmonic oscillator with only nearest neighbour interactions is recovered by choosing

$$
a_{0}=2 \kappa_{1}, \quad a_{1}=a_{N-1}=-\kappa_{1},
$$

and the remaining coefficients are set to zero.

The equations of motion for the Hamiltonian $H$ take the form

$$
\frac{d^{2}}{d t^{2}} q_{j}=\sum_{s=1}^{m} \kappa_{s}\left(q_{j+s}-2 q_{j}+q_{j-s}\right), \quad j \in \mathbb{Z} / N \mathbb{Z} .
$$


The integration is obtained by studying the dynamics in Fourier space (see e.g. [9]). In this paper we study correlations between momentum, position and local versions of energy. Following the standard procedure in the case of nearest neighbour interactions we replace the vector of position $\mathbf{q}$ by a new variable $\mathbf{r}$ so that the Hamiltonian takes the form

$$
H=\frac{1}{2}\langle\mathbf{p}, \mathbf{p}\rangle+\frac{1}{2}\langle\mathbf{r}, \mathbf{r}\rangle \text {. }
$$

Such a change of variables may be achieved by any linear transformation

$$
\mathbf{r}=T \mathbf{q}
$$

with an $N \times N$ matrix $T$ that satisfies

$$
A=T^{\top} T
$$

where $T^{\top}$ denotes the transpose of $T$. In the case of nearest neighbour interactions one may choose $r_{j}=\sqrt{\kappa_{1}}\left(q_{j+1}-q_{j}\right)$ corresponding to a circulant matrix $T$ generated by the vector $\tau=\sqrt{\kappa_{1}}(-1,1,0, \ldots, 0)$. We show in Proposition 2.2 below that short range interactions given by matrices $A$ of the form (1.3), (1.4) also admit such a localized square root. More precisely, there exists a circulant $N \times N$ matrix $T$ of the form

$$
T=\left[\begin{array}{ccccccc}
\tau_{0} & \tau_{1} & \ldots & \tau_{m} & 0 & \ldots & 0 \\
0 & \tau_{0} & \tau_{1} & \ldots & \tau_{m} & 0 & \\
& \ddots & \ddots & \ddots & \ddots & & \\
& & \ddots & \ddots & \ddots & \ddots & \\
\tau_{m} & 0 & \ddots & & & & \\
& \ddots & \ddots & \ddots & \ddots & \ddots & \ddots \\
\tau_{2} & \ldots & \tau_{m} & 0 & \ldots & \tau_{0} & \tau_{1} \\
\tau_{1} & \tau_{2} & \ldots & \tau_{m} & 0 & 0 & \tau_{0}
\end{array}\right] .
$$

that satisfies (1.6). The crucial point here is that $T$ is not the standard (symmetric) square root of the positive semidefinite matrix $A$ but a localized version generated by some vector $\boldsymbol{\tau}$ with zero entries everywhere, except possibly in the first $m+1$ components. Hence the $j$-th component of the generalized elongation $\mathbf{r}$ defined through (1.5) depends only on the components $q_{s}$ with $s=j, j+1, \ldots, j+m$. It is worth noting that $\mathbf{1}=(1, \ldots, 1)^{\top}$ satisfies $T \mathbf{1}=0$ since $\langle\mathbf{1}, A \mathbf{1}\rangle=0$. This implies

$$
\sum_{s=0}^{m} \tau_{s}=0, \quad r_{j}=\sum_{s=1}^{m} \tau_{s}\left(q_{j+s}-q_{j}\right) \quad \text { and } \quad \sum_{j=0}^{N-1} r_{j}=(1, \ldots, 1) T \mathbf{q}=0 .
$$

The local energy $e_{j}$ takes the form

$$
e_{j}=\frac{1}{2} p_{j}^{2}+\frac{1}{2} r_{j}^{2}
$$

The goal of this manuscript is to study the behaviour of the correlation functions for the momentum $p_{j}$, the generalized elongation $r_{j}$ and the local energy $e_{j}$ when $N \rightarrow \infty$ and $t \rightarrow \infty$. Due to the spatial translation invariance of the Hamiltonian $H(\mathbf{p}, \mathbf{q})=H(\mathbf{p}, \mathbf{q}+\lambda \mathbf{1})$, $\lambda \in \mathbb{R}$, that corresponds to the conservation of total momentum, we reduce the Hamiltonian system by one degree of freedom to obtain a normalizable Gibbs measure. This leads to the reduced phase space 


$$
\mathcal{M}:=\left\{(\mathbf{p}, \mathbf{q}) \in \mathbb{R}^{N} \times \mathbb{R}^{N}: \sum_{k=0}^{N-1} p_{k}=0 ; \sum_{k=0}^{N-1} q_{k}=0\right\} .
$$

We endow $\mathcal{M}$ with the Gibbs measure at temperature $\beta^{-1}$, namely:

$$
\mathrm{d} \mu=Z_{N}(\beta)^{-1} \delta\left(\sum_{k=0}^{N-1} p_{k}\right) \delta\left(\sum_{k=0}^{N-1} q_{k}\right) e^{-\beta H(\mathbf{p}, \mathbf{q})} \mathrm{d} \mathbf{p d} \mathbf{q}
$$

where $Z_{N}(\beta)$ is the norming constant and $\delta(x)$ is the delta function.

For convenience we introduce the vector

$$
\boldsymbol{u}(j, t)=\left(r_{j}(t), p_{j}(t), e_{j}(t)\right) .
$$

We consider the correlation functions

$$
S_{\alpha \alpha^{\prime}}^{N}(j, t)=\left\langle u_{\alpha}(j, t) u_{\alpha^{\prime}}(0,0)\right\rangle-\left\langle u_{\alpha}(j, t)\right\rangle\left\langle u_{\alpha^{\prime}}(0,0)\right\rangle, \quad \alpha, \alpha^{\prime}=1,2,3,
$$

where the symbol $\langle$.$\rangle refers to averages with respect to d \mu$. We calculate the limits

$$
\lim _{N \rightarrow \infty} S_{\alpha \alpha^{\prime}}^{N}(j, t)=S_{\alpha \alpha^{\prime}}(j, t) .
$$

For the harmonic oscillator with nearest-neighbour interactions such limits have been calculated in [10].

In an interesting series of papers, (see e.g. [18], and also the collection [7]) several researchers have considered the evolution of space-time correlation functions, for "anharmonic chains", which are nonlinear nearest-neighbour Hamiltonian systems of oscillators. The authors consider the deterministic evolution from random initial data sampled from a Gibbs ensemble, with a large number of particles and study the correlation functions $S_{\alpha \alpha^{\prime}}^{N}$.

In addition to intensive computational simulations [6,13], Spohn and collaborators also propose and study a nonlinear stochastic conservation law model [17,18]. Using deep physical intuition, it has been proposed that the long-time behaviour of space-time correlation functions of the deterministic Hamiltonian evolution from random initial data is equivalent to the behaviour of correlation functions of an analogous nonlinear stochastic system of PDEs. Studying this stochastic model, Spohn eventually arrives at an asymptotic description of the "sound peaks" of the correlation functions in normal modes coordinates which are related to $S_{\alpha \alpha^{\prime}}$ by orthogonal transformation:

$$
\tilde{S}_{\alpha \alpha} \cong\left(\lambda_{s} t\right)^{-2 / 3} f_{\mathrm{KPZ}}\left(\left(\lambda_{s} t\right)^{-2 / 3}(x-\alpha c t)\right),
$$

using the notation of [17, Formula (3.1)]. Here $f_{\mathrm{KPZ}}$ is a universal function that first emerges in the Kardar-Parisi-Zhang equation and it is related to the Tracy-Widom distribution, [20], (for a review see [1] and also [4]). A common element to the above cited papers is the observation that such formulae should hold for non-integrable dynamics, while the correlation functions of integrable lattices of oscillators will exhibit ballistic scaling, which means the correlation functions decay as $\frac{1}{t}$ for $t$ large. For example, in [6] the authors present the results of simulations of the Toda lattice in 3 different asymptotic regimes (the harmonic oscillator limit, the hard-particle limit, and the full nonlinear system). They present plots of the quantity $t S(x, t)$ as a function of the scaled spatial variable $x / t$ (here $S(x, t)$ represents any of the correlation functions). The numerical results support the ballistic scaling conjecture in some of the asymptotic scaling regimes. Further analysis in [19] gives a derivation of the ballistic scaling for the Toda lattice. The decay of equilibrium correlation functions show 
similar features as anomalous heat transport in one-dimensional systems $[2,3,7]$ which leads to conjecture that the two phenomena are related [8].

There is one aspect regarding all the correlations we consider that we wish to point out. For linear force laws they are oscillating rapidly in the region between the sound peaks, except for those regions that display slower decay rates (see Figs. 1 and 3). In agreement with [6] we observe numerically that these oscillations persist in the weakly nonlinear regime but disappear for fully nonlinear systems (see Figs. 4, 5).

In [12] the authors also pursue a different connection to random matrices, and in particular to the Tracy-Widom distribution. Over the last 15 years, there has emerged a story originating in the proof that for the totally asymmetric exclusion process on a 1-D lattice (TASEP), the fluctuations of the height function are governed (in a suitable limit) by the Tracy-Widom distribution. Separately, a partial differential equations model for these fluctuations emerged, which takes the form of a stochastic Burgers equation:

$$
\frac{\partial u}{\partial t}=v \frac{\partial^{2} u}{\partial x^{2}}-\lambda u \frac{\partial u}{\partial x}+\frac{\partial \zeta}{\partial x},
$$

where $\zeta$ is a stationary spatio-temporal white noise process. (The mean behaviour of TASEP is actually described by the simpler Euler equation $\frac{\partial u}{\partial t}=-\lambda u \frac{\partial u}{\partial x}$.). From these origins there have now emerged proofs, for a small collection of initial conditions, that the fluctuations of the solution to (1.12) are indeed connected to the Tracy-Widom distribution (see [1] and the references contained therein). In [12], the authors considered continuum limits of anharmonic lattices with random initial data, in which there are underlying conservation laws describing the mean behaviour that are the analogue of the Euler equation associated to (1.12). By analogy with the connection between TASEP and (1.12), they proposed that the timeintegrated currents are the analogue of the height function, and should exhibit fluctuations about their mean described by the Tracy-Widom distribution, again based on the use of the nonlinear stochastic pde system as a model for the deterministic evolution from random initial data. As one example, they consider the quantity

$$
\Phi(x, t)=\int_{0}^{t} \mathfrak{j}\left(x, t^{\prime}\right) d t^{\prime}-\int_{0}^{x} \mathfrak{u}\left(x^{\prime}, 0\right) d x^{\prime},
$$

where $\mathfrak{u}(x, t)$ arises as a sort of continuum limit of a particle system obeying a discrete analogue of a system of conservation laws taking the form $\partial_{t} \mathfrak{u}(x, t)+\partial_{x} \mathfrak{j}(x, t)=0$, in which $\mathfrak{j}(x, t)$ is a local current density for $\mathfrak{u}(x, t)$. The authors suggest a dual interpretation of $\Phi(x, t)$ as the height function from a KPZ equation, and thus arrive at the proposal that

$$
\Phi(x, t) \simeq a_{0} t+(\Gamma t)^{1 / 3} \xi_{T W},
$$

where $a_{0}$ and $\Gamma$ are model-dependent parameters, and $\xi_{T W}$ is a random amplitude with Tracy-Widom distribution.

Our main result is the analogue of the relations (1.11) for the harmonic oscillator with short range interactions and (1.14) for the harmonic oscillator.

For stating our result, we first calculate the dispersion relation $|\omega(k)|$ for the harmonic oscillator with short range interaction in the limit $N \rightarrow \infty$ obtaining

$$
f(k)=|\omega(k)|=\sqrt{2 \sum_{s=1}^{m} \kappa_{s}(1-\cos (2 \pi k s))},
$$



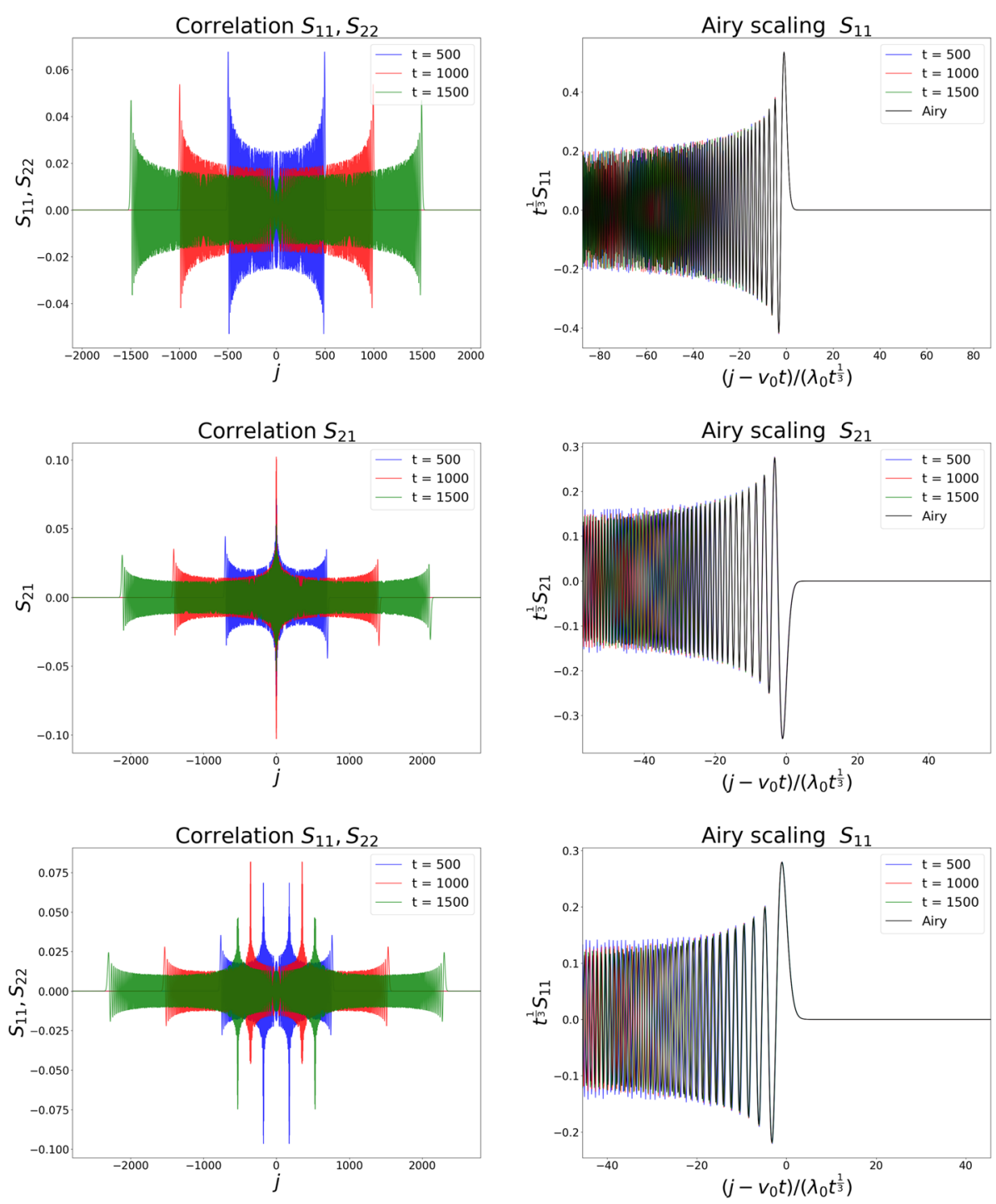

Fig. 1 Correlation functions $S_{\alpha \alpha^{\prime}}$ for the harmonic oscillator with nearest-neighbour interaction with $\kappa_{1}=1$ (top left) and the harmonic potential with $\kappa_{s}=\frac{1}{s^{2}}$ for $s=1,2$ in Example 2.8 (center left) and the potential of Example 2.9 in the bottom left. In the second column the Airy scaling (2.36) of the corresponding fastest moving peaks. The Airy asymptotic is perfectly matching the fastest peak and capturing several oscillations

see (2.21). The points $k=0,1$ contribute to the fastest moving peaks of the correlation functions that have a velocity $\pm v_{0}$ where $v_{0}=\sqrt{\sum_{s=1}^{m} s^{2} \kappa_{s}}=f^{\prime}(0) /(2 \pi)$. If $f^{\prime \prime}(k)<0$ for all $0<k \leq 1 / 2$ then as $t \rightarrow \infty$ the following holds uniformly in $j \in \mathbb{Z}$ (cf. Theorem 2.6 and Figs. 1 and 3): 

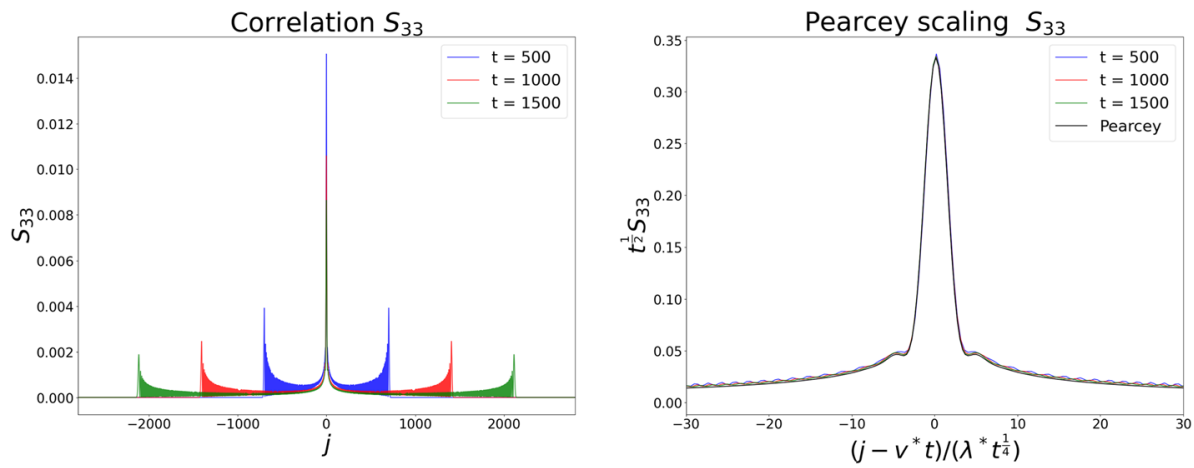

Fig. 2 Correlation function $S_{33}(j, t)$ for the potential $\kappa_{s}=1 / s^{2}$ for $m=2$ in Example 2.8 for several values of time on the left. On the right one sees that the Pearcey scaling provided in (2.45) matches perfectly for the central peak of $S_{33}(j, t)$
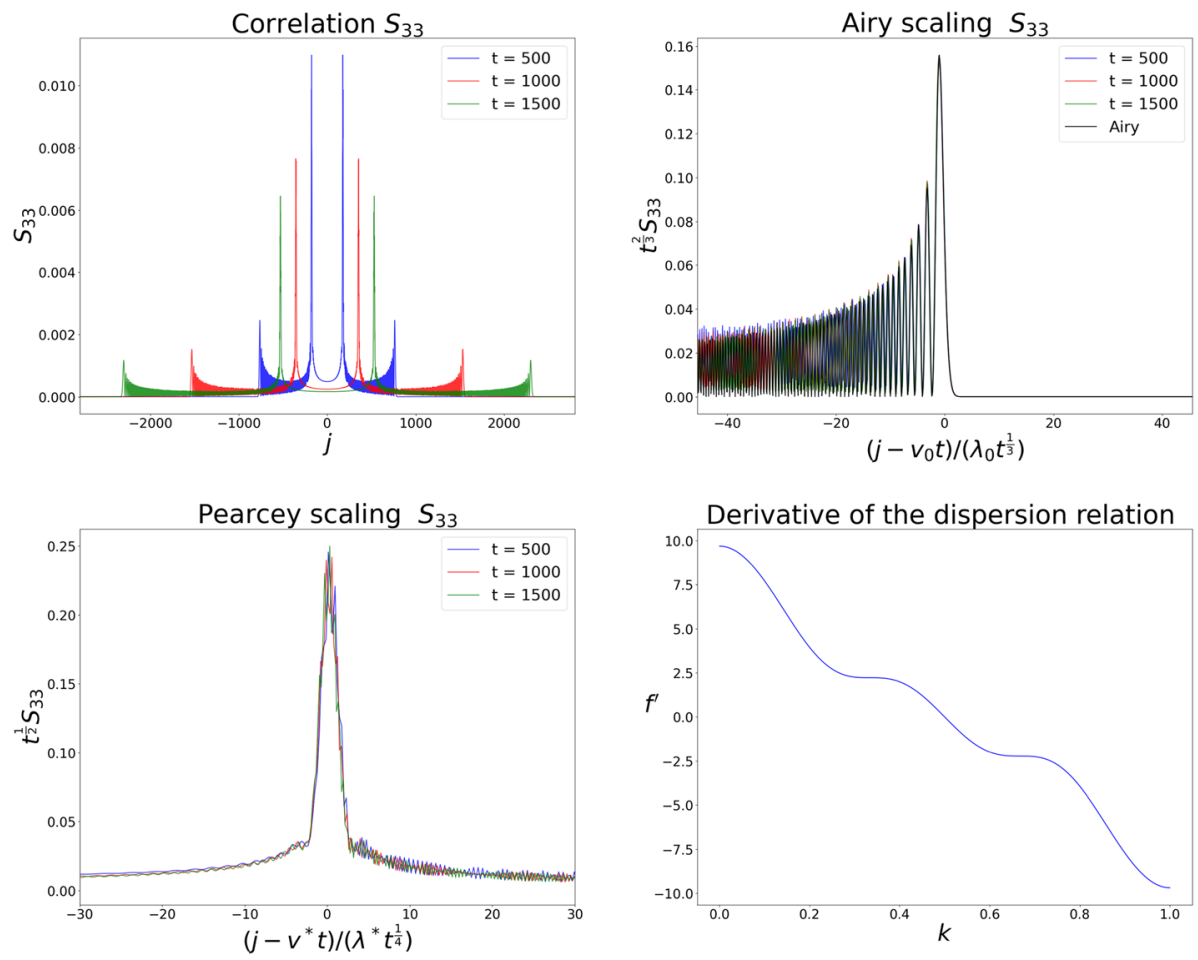

Fig. 3 Potential of Example 2.9. The top left figure displays $S_{33}(j, t)$ for several values of $t$. The scaling of $S_{33}$ according to the Airy function in Theorem 2.6 for the fastest moving peak and the scaling of the slower moving peak according to the Pearcey integral are shown top right and bottom left, respectively. The corresponding critical points of the derivative of the dispersion function can be seen in the bottom right figure 

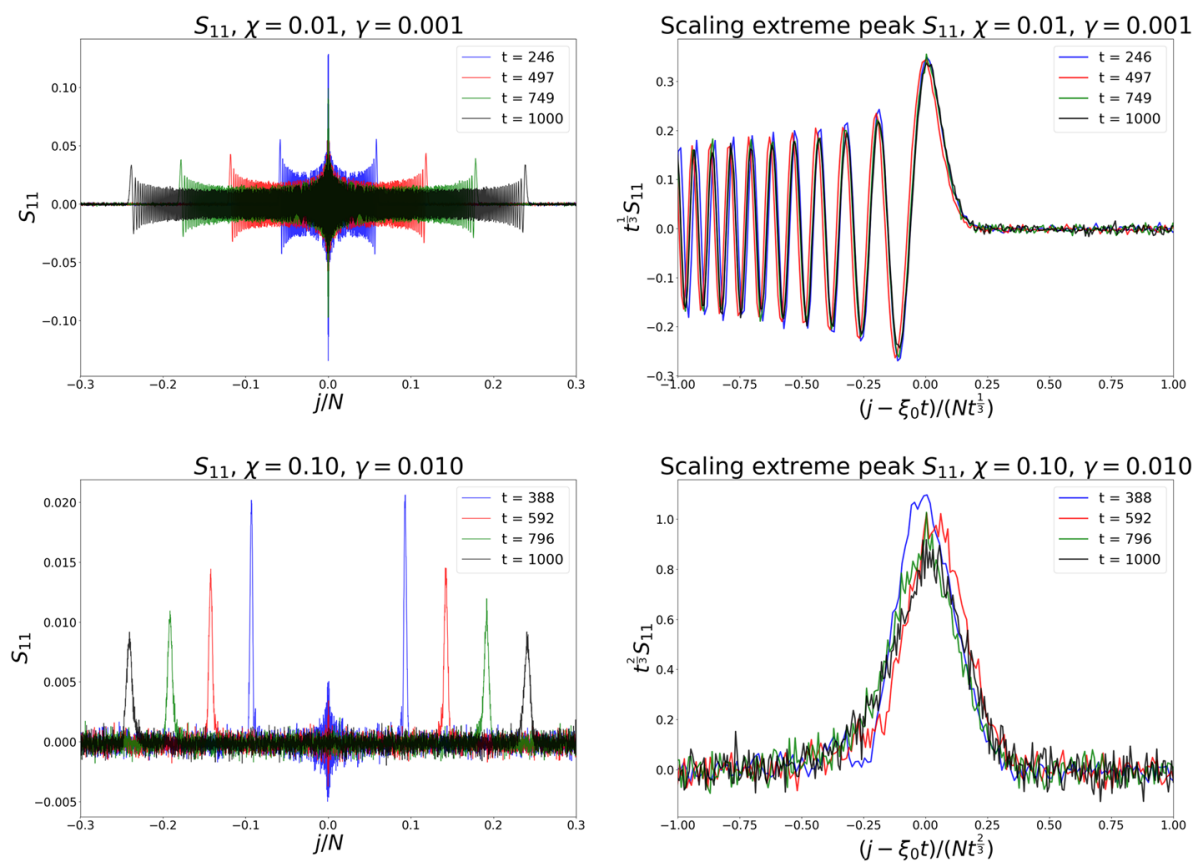

Fig. 4 Correlation function $S_{11}^{(N)}(j, t)$ for several values of times and for the perturbed Hamiltonian (3.18) with $\kappa_{S}$ as in Example 2.8, $\chi=0.01$ and $\gamma=0.001$ in the top figure and $\chi=0.1$ and $\gamma=0.01$ in the lower figure. On the right top figure, the scaling of the fastest peak according to Airy parametrix (see Theorem 2.6 and Fig. 1) and according to $t^{-2 / 3}$ in the lower figure. The speed $\xi_{0}$ of the fastest peak is determined numerically. One can see that the central peak has a low decay in the top left figure, while in the left bottom figure it is destroyed by the relatively stronger nonlinearity

$$
\begin{gathered}
S_{\alpha \alpha^{\prime}}(j, t)=\frac{1}{2 \beta \lambda_{0} t^{1 / 3}} \\
{\left[(-1)^{\alpha+\alpha^{\prime}} \mathrm{Ai}\left(\frac{j-v_{0} t}{\lambda_{0} t^{1 / 3}}\right)+\mathrm{Ai}\left(-\frac{j+v_{0} t}{\lambda_{0} t^{1 / 3}}\right)\right]+\mathcal{O}\left(t^{-1 / 2}\right), \quad \alpha, \alpha^{\prime}=1,2} \\
S_{33}(j, t)=\frac{1}{2 \beta^{2} \lambda_{0}^{2} t^{2 / 3}}\left[\mathrm{Ai}^{2}\left(\frac{j-v_{0} t}{\lambda_{0} t^{1 / 3}}\right)\right. \\
\left.+\mathrm{Ai}^{2}\left(-\frac{j+v_{0} t}{\lambda_{0} t^{1 / 3}}\right)\right]+\mathcal{O}\left(t^{-5 / 6}\right),
\end{gathered}
$$

where $\operatorname{Ai}(w)=\frac{1}{\pi} \int_{0}^{\infty} \cos \left(y^{3} / 3+w y\right) d y, w \in \mathbb{R}$, is the Airy function, and $\lambda_{0}:=$ $\frac{1}{2}\left(\frac{1}{v_{0}} \sum_{s=1}^{m} s^{4} \kappa_{s}\right)^{1 / 3}$. The above formula is the linear analogue of the Tracy-Widom distribution in (1.11).

Furthermore we can tune the spring intensities $\kappa_{s}, s=1, \ldots, m$ in (1.15) so that we can find an $(m-1)$-parameter family of potentials such that for $j \sim \pm v^{*} t$, with $0 \leq v^{*}<v_{0}$, one has

$$
S_{\alpha \alpha^{\prime}}(j, t)=\mathcal{O}\left(\frac{1}{t^{\frac{1}{4}}}\right), \quad \alpha, \alpha^{\prime}=1,2, \quad S_{33}(j, t)=\mathcal{O}\left(\frac{1}{t^{\frac{1}{2}}}\right), \quad \text { as } t \rightarrow \infty
$$



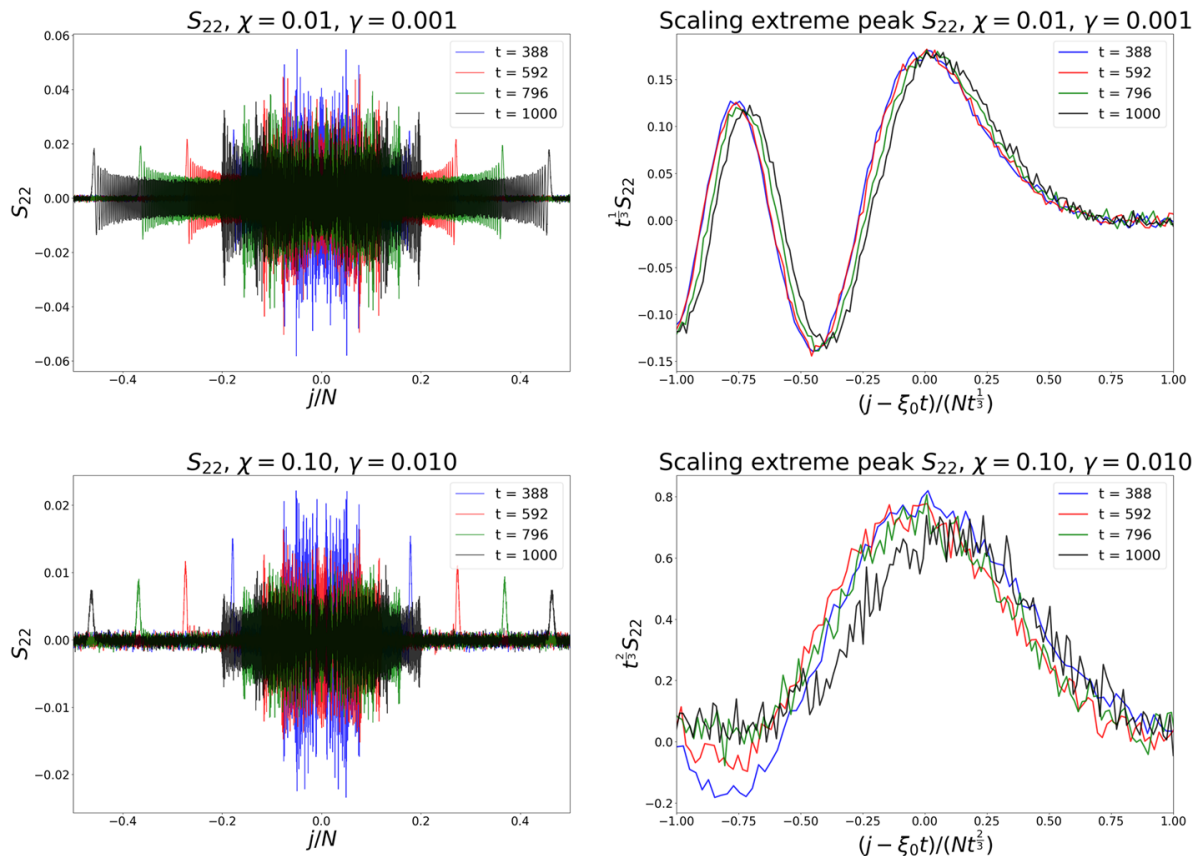

Fig. 5 Correlation function $S_{22}^{(N)}(j, t)$ for several values of times and for the perturbed Hamiltonian (3.18) with $\kappa_{S}$ as in Example 2.9, $\chi=0.01$ and $\gamma=0.001$ in the top figure and $\chi=0.1$ and $\gamma=0.01$ in the lower figure. The right top figure shows the scaling of the fastest peak compatible with the Airy parametrix and according to $t^{-2 / 3}$ in the lower figure. The speed $\xi_{0}$ of the fastest peak is determined numerically. The decay rate of the slower moving peaks that are scaling like $t^{-1 / 4}$ in the linear case (see Fig. 1 ), is not very clear due to their highly oscillatory behaviour
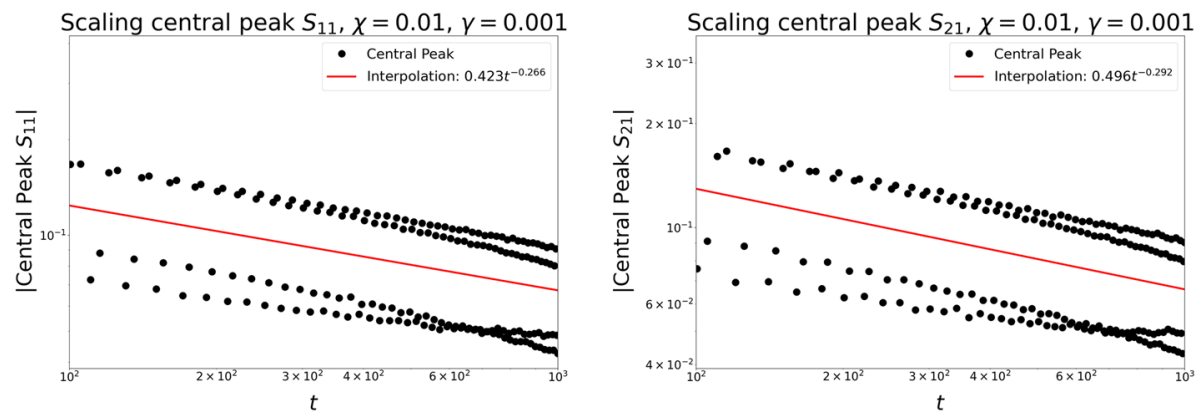

Fig. 6 Logarithmic plot of the central peak of the example in Fig. 4 for $S_{11}(j, t)$ and $S_{21}(j, t)$ and several values of times. The peak is highly oscillatory and the oscillations are interpolated by the red line that suggests a scaling of the correlation function $S_{11}(j, t)$ and $S_{21}(j, t)$ near $j \sim 0$ compatible with $t^{-\frac{1}{4}}$

In this case the local behaviour of the correlation functions is described by the Pearcey integral (see Theorem 2.7 and Figs. 2, 3). For example a potential with such behaviour is given by a spring interaction of the form $\kappa_{s}=\frac{1}{s^{2}}$ for $s=1, \ldots, m$ and $m$ even (see Example 2.8 below). 
In Sect. 3.3 we study numerically small nonlinear perturbations of the harmonic oscillator with short range interactions and our results suggest that the behaviour of the fastest peak has a transition from the Airy asymptotic (1.16) to the Tracy-Widom asymptotic (1.11), depending on the strength of the nonlinearity. Namely the asymptotic behaviour in (1.11) that has been conjectured for nearest-neighbour interactions seems to persist also for sufficiently strong nonlinear perturbations of the harmonic oscillator with short range interactions. Remarkably, our numerical simulations indicate that the non generic decay in time of other peaks in the correlation functions persists under small nonlinear perturbations with the same power law $t^{-1 / 4}$ as in the linear case, see e.g. Figs. 4 and 6.

So as not to overlook a large body of related work, we observe that the quantities we consider here are somewhat different than those considered in the study of thermal transport, though there is of course overlap. (We refer to the Lecture Notes [7] for an overview of this research area and also the seminal paper [15].) As mentioned above, we study the dynamical evolution of space-time correlation functions and the statistical description of random height functions, where the only randomness comes from the initial data. By comparison, in the consideration of heat conduction and transport in low dimensions, anharmonic chains are often connected at their ends to heat reservoirs of different temperatures, and randomness is present primarily in the dynamical laws, not only in fluctuations of initial data.

This manuscript is organized as follows. In Sect. 2 we study the harmonic oscillator with short range interactions and we introduce the necessary notation and the change of coordinates $\mathbf{q} \rightarrow \mathbf{r}$ that enables us to study correlation functions. We then study the time decay of the correlation functions via steepest descent analysis and we show that the two fastest peaks travelling in opposite directions originate from the points $k=0$ and $k=1$ in the spectrum. Such peaks have a decay described by the Airy scaling. We then show the existence of potentials such that the correlation functions have a slower time decaying with respect to "Airy peaks". In Sect. 3 we show that the harmonic oscillator with short range interactions has a complete set of local integrals of motion in involution and the correlation functions of such integrals have the same structure as the energy-energy correlation function. Finally, we show that the evolution equations for the generalized position, momentum can be written in the form of conservation laws which have a potential function. For the case of the harmonic oscillator with nearest-neighbour interaction, we show that this function is a Gaussian random variable and determine the leading order behaviour of its variance as $t \rightarrow \infty$. This may be viewed as the analogue of formula (1.14) for the linear case. Technicalities and a description of our numerics are deferred to the Appendix.

\section{The Harmonic Oscillator with Short Range Interactions}

As it was explained in the introduction we rewrite the Hamiltonian for the harmonic oscillator with short range interactions

$$
H(\mathbf{p}, \mathbf{q})=\sum_{j=0}^{N-1} \frac{p_{j}^{2}}{2}+\sum_{s=1}^{m} \frac{\kappa_{s}}{2} \sum_{j=0}^{N-1}\left(q_{j}-q_{j+s}\right)^{2}=\sum_{j=0}^{N-1}\left(\frac{p_{j}^{2}}{2}+\frac{1}{2}\left(\sum_{s=1}^{m} \tau_{s}\left(q_{j+s}-q_{j}\right)\right)^{2}\right)
$$

so that we may define a Hamiltonian density

$$
e_{j}=\frac{p_{j}^{2}}{2}+\frac{1}{2}\left(\sum_{s=1}^{m} \tau_{s}\left(q_{j+s}-q_{j}\right)\right)^{2}
$$


which is local in the variables $(\mathbf{p}, \mathbf{q})$ for fixed $m$. Namely, if we let $N \rightarrow \infty$, the quantity $e_{j}$ involves a finite number of physical variables $(\mathbf{p}, \mathbf{q})$. Recall that the coefficients $\tau_{s}$ are the entries of the circulant localized square root $T$ of the matrix $A$ by which we mean a solution of the equation (1.6) of the form (1.7). The matrix $T$ will also play a role in constructing a complete set of integrals that have a local density in the sense that we just described for the energy.

In order to state our result we have to introduce some notation. First of all, a matrix $A$ of the form (1.3) with $\mathbf{a} \in \mathbb{R}^{N}$ is called a circulant matrix generated by the vector $\mathbf{a}$.

Definition 2.1 ( $m$-physical vector and half- $m$-physical vector) Fix $m \in \mathbb{N}$. For any odd $N>$ $2 m$, a vector $\tilde{\mathbf{x}} \in \mathbb{R}^{N}$ is said to be $m$-physical generated by $\mathbf{x}=\left(x_{0}, x_{1}, \ldots, x_{m}\right) \in \mathbb{R}^{m+1}$ if $x_{0}=-2 \sum_{s=1}^{m} x_{s}$ and

$$
\begin{aligned}
& \tilde{x}_{0}=x_{0}, \\
& \tilde{x}_{1}=\tilde{x}_{N-1}=x_{1}<0, \quad \tilde{x}_{m}=\tilde{x}_{N-m}=x_{m}<0, \\
& \tilde{x}_{k}=\tilde{x}_{N-k}=x_{k} \leq 0, \text { for } 1<k<m, \\
& \tilde{x}_{k}=0, \text { otherwise, }
\end{aligned}
$$

while the vector $\tilde{\mathbf{x}} \in \mathbb{R}^{N}$ is called half-m-physical generated by $\mathbf{y} \in \mathbb{R}^{m+1}$ if $y_{0}=-\sum_{s=1}^{m} y_{s}$ and

$$
\begin{aligned}
& \tilde{x}_{k}=y_{k}, \text { for } 0 \leq k \leq m \\
& \tilde{x}_{k}=0, \text { for } m<k \leq N-1 .
\end{aligned}
$$

Following the proof of a classic lemma by Fejér and Riesz, see e.g. [16, pg. 117 f], one can show that a circulant symmetric matrix $A$ of the form (1.2) generated by a $m$-physical vector a always has a circulant localized square root $T$ that is generated by a half- $m$ physical vector $\tau$.

Proposition 2.2 Fix $m \in \mathbb{N}$. Let the circulant matrix A be generated by an m-physical vector a, then there exist a circulant matrix $T$ generated by an half-m-physical vector $\boldsymbol{\tau}$ such that:

$$
A=T^{\top} T .
$$

Moreover, we can choose $\tau$ such that $\sum_{s=1}^{m} s \tau_{s}>0$. Then one has $\sum_{s=1}^{m} s \tau_{s}=\sqrt{\sum_{s=1}^{m} s^{2} \kappa_{s}}$.

The proof of the proposition is contained in Appendix A:.

For example, if we consider $m=1$, and $a_{0}=2 \kappa_{1}$ and $a_{1}=a_{N-1}=-\kappa_{1}$. The matrix $T$ is generated by the vector $\tau=\left(\tau_{0}, \tau_{1}\right)$ with $\tau_{0}=-\sqrt{\kappa_{1}}$ and $\tau_{1}=\sqrt{\kappa_{1}}$. When $m=2$ and $a_{0}=2 \kappa_{1}+2 \kappa_{2}, a_{1}=a_{N-1}=-\kappa_{1}, a_{2}=a_{N-2}=-\kappa_{2}$. The matrix $T$ is generated by the vector $\boldsymbol{\tau}=\left(\tau_{0}, \tau_{1}, \tau_{2}\right)$ with

$$
\begin{aligned}
& \tau_{0}=-\frac{\sqrt{\kappa_{1}}}{2}-\frac{1}{2} \sqrt{\kappa_{1}+4 \kappa_{2}}, \quad \tau_{1}=\sqrt{\kappa_{1}}, \\
& \tau_{2}=-\frac{\sqrt{\kappa_{1}}}{2}+\frac{1}{2} \sqrt{\kappa_{1}+4 \kappa_{2}},
\end{aligned}
$$

so that the quantities $r_{j}$ are defined as

$$
r_{j}=\tau_{1}\left(q_{j+1}-q_{j}\right)+\tau_{2}\left(q_{j+2}-q_{j}\right), \quad j \in \mathbb{Z} / N \mathbb{Z} .
$$

Next we integrate the equation of motions. The Hamiltonian $H(\mathbf{p}, \mathbf{q})$ represents clearly an integrable system that can be integrated passing through Fourier transform. Let $\mathcal{F}$ be the 
discrete Fourier transform with entries $\mathcal{F}_{j, k}:=\frac{1}{\sqrt{N}} e^{-2 \mathrm{i} \pi j k / N}$ with $j, k=0, \ldots, N-1$. It is immediate to verify that

$$
\mathcal{F}^{-1}=\overline{\mathcal{F}} \quad \mathcal{F}^{\top}=\mathcal{F}
$$

Thanks to the above properties, the transformation defined by

$$
(\widehat{p}, \widehat{q})=(\overline{\mathcal{F}} p, \mathcal{F} q)
$$

is canonical. Furthermore $\overline{\widehat{p}}_{j}=\widehat{p}_{N-j}$ and $\overline{\widehat{q}}_{j}=\widehat{q}_{N-j}$, for $j=1, \ldots, N-1$, while $\widehat{p}_{0}$ and $\widehat{q}_{0}$ are real variables. The matrices $T$ and $A$ are circulant matrices and so they are reduced to diagonal form by $\mathcal{F}$ :

$$
\mathcal{F} A \mathcal{F}^{-1}=\mathcal{F} T^{\top} T \mathcal{F}^{-1}=\overline{\left(\mathcal{F} T \mathcal{F}^{-1}\right)}{ }^{\top}\left(\mathcal{F} T \mathcal{F}^{-1}\right) .
$$

Let $\omega_{j}$ denote the eigenvalues of the matrix $T$ ordered so that $\mathcal{F} T \mathcal{F}^{-1}=\operatorname{diag}\left(\omega_{j}\right)$. Then $\left|\omega_{j}\right|^{2}$ are the (non negative) eigenvalues of the matrix $A$ and

$$
\left|\omega_{j}\right|^{2}=\sqrt{N}(\overline{\mathcal{F}} \tilde{\mathbf{a}})_{j}, \quad \omega_{j}=\sqrt{N}(\overline{\mathcal{F}} \tilde{\boldsymbol{\tau}})_{j}, \quad j=0, \ldots, N-1,
$$

where $\tilde{\mathbf{a}}$ is the $m$-physical vector generated by a and $\tilde{\boldsymbol{\tau}}$ is the half $m$-physical vector generated by $\boldsymbol{\tau}$ according to Definition 2.1. It follows that

$$
\omega_{0}=0, \quad \omega_{j}=\bar{\omega}_{N-j}, \quad j=1, \ldots, N-1,
$$

which implies $\left|\omega_{j}\right|^{2}=\left|\omega_{N-j}\right|^{2}, j=1, \ldots, N-1$. The Hamiltonian $H$, can be written as the sum of $N-1$ oscillators

$$
H(\widehat{\mathbf{p}}, \widehat{\mathbf{q}})=\frac{1}{2}\left(\sum_{j=1}^{N-1}\left|\widehat{p}_{j}\right|^{2}+\left|\omega_{j}\right|^{2}\left|\widehat{q}_{j}\right|^{2}\right)=\sum_{j=1}^{\frac{N-1}{2}}\left|\widehat{p}_{j}\right|^{2}+\left|\omega_{j}\right|^{2}\left|\widehat{q}_{j}\right|^{2} .
$$

There are no terms involving $\widehat{p}_{0}, \widehat{q}_{0}$ since the conditions defining $\mathcal{M}(1.8)$ imply that $\widehat{p}_{0}=0$ and $\widehat{q}_{0}=0$. The Hamilton equations are

$$
\left\{\begin{array}{l}
\frac{d}{d t} \widehat{q}_{j}={\widehat{p_{j}}} \\
\frac{d}{d t} \widehat{p}_{j}=-\left|\omega_{j}\right|^{2} \widehat{q}_{j} .
\end{array}\right.
$$

Thus the general solution reads:

$$
\begin{aligned}
& \widehat{q}_{j}(t)=\widehat{q}_{j}(0) \cos \left(\left|\omega_{j}\right| t\right)+\frac{\overline{\widehat{p}_{j}(0)}}{\left|\omega_{j}\right|} \sin \left(\left|\omega_{j}\right| t\right), \\
& \overline{\widehat{p}_{j}(t)}=\overline{\widehat{p}_{j}(0)} \cos \left(\left|\omega_{j}\right| t\right)-\left|\omega_{j}\right| \widehat{q}_{j}(0) \sin \left(\left|\omega_{j}\right| t\right), \quad j=1, \ldots, N-1,
\end{aligned}
$$

and $\widehat{q}_{0}(t)=0$ and $\widehat{p}_{0}(t)=0$. Inverting the Fourier transform, we recover the variables $\mathbf{q}=\mathcal{F}^{-1} \widehat{\mathbf{q}}, \mathbf{p}=\mathcal{F} \widehat{\mathbf{p}}$ and $\mathbf{r}=\mathcal{F}^{-1} \widehat{\mathbf{r}}$ where

$$
\widehat{r}_{j}=\omega_{j} \widehat{q}_{j}, j=0, \ldots, N-1 \text {. }
$$




\section{Correlation Decay}

We now study the decay of correlation functions for Hamiltonian systems of the form (1.2). We recall the definition (1.9) of the Gibbs measure at temperature $\beta^{-1}$ on the reduced phase space $\mathcal{M}$, namely:

$$
\mathrm{d} \mu=Z_{N}(\beta)^{-1} \delta\left(\sum_{k=0}^{N-1} p_{k}\right) \delta\left(\sum_{k=0}^{N-1} q_{k}\right) e^{-\beta H(\mathbf{p}, \mathbf{q})} \mathrm{d} \mathbf{p d} \mathbf{q}
$$

where $Z_{N}(\beta)$ is the norming constant of the probability measure. For a function $f=f(\mathbf{p}, \mathbf{q})$ we define its average as

$$
\langle f\rangle:=\int_{\mathbb{R}^{2 N}} f(\mathbf{p}, \mathbf{q}) \mathrm{d} \mu .
$$

We first compute all correlation functions (1.10), then we will evaluate the limit $N \rightarrow \infty$. We first observe that (1.9) in the variables $(\widehat{\mathbf{p}}, \widehat{\mathbf{q}}):=(\overline{\mathcal{F}} \mathbf{p}, \mathcal{F} \mathbf{q})$ becomes

$$
\mathrm{d} \mu=Z_{N}(\beta)^{-1} \prod_{j=1}^{\frac{N-1}{2}} e^{-\beta\left(\left|\widehat{p}_{j}\right|^{2}+\left|\omega_{j}\right|^{2}\left|\widehat{q}_{j}\right|^{2}\right)} \mathrm{d} \widehat{p}_{j} \mathrm{~d} \widehat{q}_{j}
$$

where $\mathrm{d} \widehat{p}_{j} \mathrm{~d} \widehat{q}_{j}=\mathrm{d} \Re \widehat{p}_{j} \mathrm{~d} \Im \widehat{p}_{j} \mathrm{~d} \Re \widehat{q}_{j} \mathrm{~d} \Im \widehat{q}_{j}$ and we recall that $\widehat{p}_{j}=\widehat{p}_{N-j}, \widehat{q}_{j}=\widehat{q}_{N-j}, \widehat{r}_{j}=$ $\omega_{j} \widehat{q}_{j}$, for $j=1, \ldots, N-1$.

From the evolution of $\widehat{p}_{j}$ and $\widehat{q}_{j}$ in (2.8) and (2.9), we arrive at the relations

$$
\begin{aligned}
&\left\langle\widehat{p}_{j}(t) \overline{\widehat{p}_{k}(0)}\right\rangle=\left\langle\overline{\widehat{p}_{k}(0)}\left(\widehat{p}_{j}(0) \cos \left(\left|\omega_{j}\right| t\right)-\left|\omega_{j}\right| \overline{\widehat{q}_{j}(0)} \sin \left(\left|\omega_{j}\right| t\right)\right)\right\rangle=\delta_{j, k} \frac{1}{\beta} \cos \left(\left|\omega_{j}\right| t\right) \\
&\left\langle\widehat{p}_{j}(t) \widehat{r}_{k}(0)\right\rangle=\left\langle\omega_{k} \widehat{q}_{k}(0)\left(\widehat{p}_{j}(0) \cos \left(\left|\omega_{j}\right| t\right)-\left|\omega_{j}\right| \overline{\hat{q}_{j}(0)} \sin \left(\left|\omega_{j}\right| t\right)\right)\right\rangle \\
&=-\delta_{j, k} \frac{\omega_{j}}{\left|\omega_{j}\right| \beta} \sin \left(\left|\omega_{j}\right| t\right), \\
&\left\langle\widehat{r}_{j}(t) \widehat{p}_{k}(0)\right\rangle=\left\langle\omega_{j} \widehat{p}_{k}(0)\left(\widehat{q}_{j}(0) \cos \left(\left|\omega_{j}\right| t\right)+\frac{\overline{\widehat{p}_{j}(0)}}{\left|\omega_{j}\right|} \sin \left(\left|\omega_{j}\right| t\right)\right)\right\rangle=\delta_{j, k} \frac{\omega_{j}}{\left|\omega_{j}\right| \beta} \sin \left(\left|\omega_{j}\right| t\right) \\
&\left\langle\widehat{r}_{j}(t) \overline{\widehat{r}_{k}(0)}\right\rangle=\left\langle\bar{\omega}_{k} \omega_{j} \widehat{\widehat{q}}_{k}(0)\right. \\
&
\end{aligned}
$$

Now we are ready to compute explicitly the correlation functions in the physical variables. We show the computation for the case $S_{11}^{N}(j, t)$, and we leave to the reader the details for the other cases:

$$
\begin{aligned}
S_{11}^{N}(j, t) & =\left\langle r_{j}(t) r_{0}(0)\right\rangle=\frac{1}{N}\left\langle\sum_{k, l=1}^{N-1} \widehat{r}_{k}(t) \widehat{r}_{l}(0) e^{2 \pi l} \frac{j k}{N}\right\rangle \\
& =\frac{1}{N \beta} \sum_{l=1}^{N-1} \cos \left(\left|\omega_{l}\right| t\right) \cos \left(2 \pi \frac{l j}{N}\right)=S_{22}^{N}(j, t)
\end{aligned}
$$


In the same way we have that:

$$
\begin{aligned}
& S_{12}^{N}(j, t)=\frac{1}{N \beta} \sum_{l=1}^{N-1} \sin \left(\left|\omega_{l}\right| t\right) \cos \left(2 \pi \frac{l j}{N}+\arg \left(\omega_{l}\right)\right) \\
& S_{21}^{N}(j, t)=-\frac{1}{N \beta} \sum_{l=1}^{N-1} \sin \left(\left|\omega_{l}\right| t\right) \cos \left(2 \pi \frac{l j}{N}-\arg \left(\omega_{l}\right)\right) \\
& S_{31}^{N}(j, t)=S_{32}^{N}(j, t)=S_{13}^{N}(j, t)=S_{23}^{N}(j, t)=0 \\
& S_{33}^{N}(j, t)=\frac{1}{2}\left(\left(S_{11}^{N}\right)^{2}+\left(S_{22}^{N}\right)^{2}+\left(S_{12}^{N}\right)^{2}+\left(S_{21}^{N}\right)^{2}\right)+\frac{3(N-1)}{2 N^{2} \beta^{2}} .
\end{aligned}
$$

The dispersion relation given by (2.4) takes the form

$$
\begin{aligned}
& \omega_{\ell}=-\sum_{s=1}^{m} \tau_{s}\left(1-\cos \left(2 \pi \frac{s \ell}{N}\right)\right)+\mathrm{i} \sum_{s=1}^{m} \tau_{s} \sin \left(2 \pi \frac{s \ell}{N}\right) \\
& \left|\omega_{\ell}\right|^{2}=\sum_{s=0}^{N-1} a_{s} e^{-2 \pi \mathrm{i} \frac{s \ell}{N}}=2 \sum_{s=1}^{m} \kappa_{s}\left(1-\cos \left(2 \pi \frac{s \ell}{N}\right)\right),
\end{aligned}
$$

where we substitute for the $a_{s}$ their values as in (1.4). We are interested in obtaining the continuum limit of the above correlation functions. We first define $\omega(k)$ to provide continuum limits of $\omega_{\ell}$ and $\left|\omega_{\ell}\right|^{2}$, namely

$$
\begin{aligned}
\omega(k) & :=-\sum_{s=1}^{m} \tau_{s}(1-\cos (2 \pi s k))+\mathrm{i} \sum_{s=1}^{m} \tau_{s} \sin (2 \pi s k) \\
|\omega(k)|^{2} & =2 \sum_{s=1}^{m} \kappa_{s}(1-\cos (2 \pi k s)),
\end{aligned}
$$

where the variable $\ell / N$ has been approximated with $k \in[0,1]$. One may use equation (A.1) to check the consistency of the two equations of (2.21). To this end observe that $\omega(k)=Q\left(e^{-2 \pi i k}\right), \overline{\omega(k)}=Q\left(e^{2 \pi i k}\right)$, and $|\omega(k)|^{2}=\ell\left(e^{2 \pi i k}\right)$.

Lemma 2.3 Let $\omega(k)$ be defined as in (2.21), set $f(k):=|\omega(k)|$, and denote $\theta(k):=$ $\arg (\omega(k))$ for $0 \leq k \leq 1$, where the ambiguity in the definition of $\theta$ is settled by requiring $\theta$ to be continuous with $\theta(0) \in(-\pi, \pi]$. Then, for all $k \in[0,1]$ we have

$$
\begin{aligned}
\omega(1-k) & =\overline{\omega(k)}, \\
f(1-k) & =f(k), \\
\theta(1-k) & \equiv-\theta(k) \quad(\bmod 2 \pi) .
\end{aligned}
$$

Furthemore, the functions $f$ and $\theta-\frac{\pi}{2}$ are $C^{\infty}$ on $[0,1]$ and they both possess odd $C^{\infty}$. extensions at $k=0$ which implies in particular $\theta(0)=\frac{\pi}{2}$.

Proof The symmetries follow directly from the definition of $\omega$ in (2.21). From (2.21) we also learn that $|\omega(k)|^{2} \geq 2 \kappa_{1}(1-\cos (2 \pi k))>0$ for $k \in(0,1)$. Thus the smoothness of $f$ and $\theta$ only needs to be investigated for $k \in\{0,1\}$. By symmetry we only need to study the case $k=0$. The smoothness of the function $\theta$ may be obtained from the expansion near $k=0$

$$
\cot (\theta(k))=-k \pi \frac{\sum_{s=1}^{m} s^{2} \tau_{s}}{\sum_{s=1}^{m} s \tau_{s}}+\mathcal{O}\left(k^{3}\right)
$$


together with $\sum_{s=1}^{m} s \tau_{s}>0$ (see Proposition 2.2). Since $\cot (\theta(0))=0$ and $\Im \omega(k)>0$ for small positive values of $k$ we conclude that $\theta(0)=\frac{\pi}{2}$ from the requirement $\theta(0) \in(-\pi, \pi]$. This also implies the existence of a smooth odd extension of $\theta-\frac{\pi}{2}$ at $k=0$ because $\cot (\theta(k))$ has such an extension. For the function $f$ the claims follow from the representation

$$
f(k)=2 \pi k\left(\sum_{s=1}^{m} s^{2} \kappa_{s} \operatorname{sinc}^{2}(\pi s k)\right)^{1 / 2}
$$

near $k=0$ where $\operatorname{sinc}(x)=\frac{\sin (x)}{x}$ denotes the smooth and even sinus cardinalis function.

Lemma 2.4 In the limit $N \rightarrow \infty$ the correlation functions have the following expansion

$$
\begin{aligned}
& S_{\alpha \alpha^{\prime}}^{N}(j, t)+\frac{\delta_{\alpha \alpha^{\prime}}}{N \beta}=S_{\alpha \alpha^{\prime}}(j, t)+\mathcal{O}\left(N^{-\infty}\right), \quad \alpha, \alpha^{\prime}=1,2, \\
& S_{33}^{N}(j, t)=S_{33}(j, t)+\mathcal{O}\left(N^{-1}\right),
\end{aligned}
$$

where $\delta_{\alpha \alpha^{\prime}}$ denotes the Kronecker delta,

$$
\begin{aligned}
& S_{11}(j, t)=S_{22}(j, t)=\frac{1}{\beta} \int_{0}^{1} \cos (|\omega(k)| t) \cos (2 \pi k j) \mathrm{d} k \\
& S_{12}(j, t)=\frac{1}{\beta} \int_{0}^{1} \sin (|\omega(k)| t) \cos (2 \pi k j+\theta(k)) \mathrm{d} k, \\
& S_{21}(j, t)=-\frac{1}{\beta} \int_{0}^{1} \sin (|\omega(k)| t) \cos (2 \pi k j-\theta(k)) \mathrm{d} k, \\
& S_{33}(j, t)=\frac{1}{2}\left(S_{11}^{2}+S_{22}^{2}+S_{12}^{2}+S_{21}^{2}\right),
\end{aligned}
$$

and $\theta(k)=\arg \omega(k)$ with $\omega(k)$ as in (2.21).

Proof For any periodic $C^{\infty}$-function $g$ on the real line with period $1, g(k)=\sum_{n \in \mathbb{Z}} \hat{g}_{n} e^{2 \pi i k n}$, one has

$$
\frac{1}{N} \sum_{\ell=0}^{N-1} g\left(\frac{\ell}{N}\right)=\sum_{m \in \mathbb{Z}} \hat{g}_{m N}=\int_{0}^{1} g(k) \mathrm{d} k+\mathcal{O}\left(N^{-\infty}\right) .
$$

It follows from Lemma 2.3 that the integrands in (2.25)-(2.27) can be extended to 1-periodic smooth functions because we have for small positive values of $k$ that

$$
\begin{aligned}
\cos (f(-k) t) \cos (-2 \pi k j) & =\cos (f(k) t) \cos (-2 \pi k j) \\
& =\cos (f(1-k) t) \cos (2 \pi(1-k) j), \\
\sin (f(-k) t) \cos (-2 \pi k j \pm \theta(-k)) & =-\sin (f(k) t) \cos (-2 \pi k j \pm(\pi-\theta(k))) \\
& =\sin (f(1-k) t) \cos (2 \pi(1-k) j \pm \theta(1-k)) .
\end{aligned}
$$

Observing in addition that the summands corresponding to $\ell=0$ are missing in (2.15)-(2.17) the first claim is proved. Together with (2.19) this also implies the second claim.

Next we analyse the leading order behaviour (as $t \rightarrow \infty$ ) of the limiting correlation functions $S_{\alpha \alpha^{\prime}}(j, t)$ using the method of steepest descent. In order to explain the phenomena that may occur we start by discussing $S_{11}$. Denote

$$
\xi:=\frac{j}{t} \quad \text { and } \quad \phi_{ \pm}(k, \xi):=f(k) \pm 2 \pi \xi k
$$


With these definitions and using the symmetry (2.23) we may write

$$
S_{11}(j, t)=\frac{1}{2 \beta} \Re \int_{0}^{1}\left(e^{i t(f(k)+2 \pi \xi k)}+e^{i t(f(k)-2 \pi \xi k)}\right) d k=\frac{1}{\beta} \Re \int_{0}^{1} e^{i t \phi_{-}(k, \xi)} d k
$$

The leading order behaviour $(t \rightarrow \infty)$ of such an integral is determined by the stationary phase points $k_{0} \in[0,1]$, i.e. by the solutions of the equation $\frac{\partial}{\partial k} \phi_{-}\left(k_{0}, \xi\right)=0$ which depend on the value of $\xi$.

Such stationary phase points do not need to exist. In fact, as we see in Lemma 2.5(b) below, the range of $f^{\prime}$ is given by some interval $\left[-2 \pi v_{0}, 2 \pi v_{0}\right]$ so that there are no stationary phase points for $|\xi|>v_{0}$. As in the proof of Lemma 2.4 one can argue that the integrand $\Re e^{i t \phi_{-}(k, j / t)}$ can be extended to a periodic smooth function of $k$ on the real line with period 1 . It then follows from integration by parts that $S_{11}(j, t)$ decays rapidly in time. More precisely, for every fixed $\delta>0$ we have

$$
S_{11}(j, t)=\mathcal{O}\left(t^{-\infty}\right) \quad \text { as } t \rightarrow \infty, \text { uniformly for }|j| \geq\left(v_{0}+\delta\right) t .
$$

This justifies the name of sound speed for the quantity $v_{0}$.

In the case $|\xi| \leq v_{0}$ there always exists at least one stationary phase point $k_{0}=k_{0}(\xi) \in$ $[0,1]$. Each stationary phase point may provide an additive contribution to the leading order behaviour of $\int_{0}^{1} e^{i t \phi_{-}(k, j / t)} d k$ for $j$ near $\xi t$. However, the order of the contribution depends on the multiplicity of the stationary phase point. For example, let $k_{0}$ be a stationary phase point of $\phi_{-}(\cdot, \xi)$, i.e. $\frac{\partial}{\partial k} \phi_{-}\left(k_{0}, \xi\right)=0$. Denote by $\ell$ the smallest integer bigger than 1 for which $\frac{\partial^{\ell}}{\partial k^{\ell}} \phi_{-}\left(k_{0}, \xi\right) \neq 0$. Then $k_{0}$ contributes a term of order $t^{1 / \ell}$ to the $t$-asymptotics of $\int_{0}^{1} e^{i t \phi_{-}(k, j / t)} d k$ for $j$ in a suitable neighbourhood of $\xi t$.

Before treating the general situation let us recall the case of nearest-neighbour interactions. There we have

$$
f(k)=f_{1}(k)=\sqrt{2 \kappa_{1}(1-\cos (2 \pi k))}=2 \sqrt{\kappa_{1}} \sin (\pi k), \quad k \in[0,1] .
$$

The range of $f_{1}^{\prime}$ equals $\left[-2 \pi v_{0}, 2 \pi v_{0}\right]$ with $v_{0}=\sqrt{\kappa_{1}}$. For every $|\xi| \leq v_{0}$ there exists exactly one stationary phase point $k_{0}(\xi) \in[0,1]$ of $\phi_{-}(\cdot, \xi)$ that is determined by the relation $\cos \left(\pi k_{0}(\xi)\right)=\xi / v_{0}$. A straight forward calculation gives

$$
\frac{\partial^{2}}{\partial k^{2}} \phi_{-}\left(k_{0}(\xi), \xi\right)=f_{1}^{\prime \prime}\left(k_{0}(\xi)\right)=-2 \pi^{2} \sqrt{v_{0}^{2}-\xi^{2}}=0 \Leftrightarrow \xi= \pm v_{0} .
$$

Moreover, we have $k_{0}\left(v_{0}\right)=0$ and $k_{0}\left(-v_{0}\right)=1$ and therefore $\frac{\partial^{3}}{\partial k^{3}} \phi_{-}\left(k_{0}\left( \pm v_{0}\right), \pm v_{0}\right)=$ $\mp 2 \pi^{3} v_{0} \neq 0$. This implies that in addition to (2.31) we have $S_{11}(j, t)=\mathcal{O}\left(t^{-1 / 2}\right)$, except for $j$ near $\pm v_{0} t$ where $S_{11}(j, t)=\mathcal{O}\left(t^{-1 / 3}\right)$. In order to determine the behaviour near the least decaying peaks that travel at speeds $\pm v_{0}$ we expand $f_{1}$ near the stationary phase points. Let us first consider $\xi=v_{0}$ with $k_{0}=0$. Introducing $\lambda_{0}=\frac{1}{2 \pi}\left|f_{1}^{\prime \prime \prime}(0) / 2\right|^{1 / 3}=\frac{1}{2} v_{0}^{1 / 3}$ we obtain

$$
f_{1}(k)=2 \pi v_{0} k-\frac{1}{3}\left(2 \pi \lambda_{0} k\right)^{3}+\mathcal{O}\left(k^{5}\right), \quad \text { as } k \rightarrow 0 .
$$

Substituting $y=2 \pi \lambda_{0} t^{1 / 3} k$ leads for $k$ close to 0 to the asymptotic expression

$$
t \phi_{-}(k, j / t)=\frac{v_{0} t-j}{\lambda_{0} t^{1 / 3}} y-\frac{1}{3} y^{3}+\mathcal{O}\left(t^{-2 / 3}\right), \quad \text { as } t \rightarrow \infty .
$$


Using the well-known representation $\operatorname{Ai}(w)=\frac{1}{\pi} \int_{0}^{\infty} \cos \left(y^{3} / 3+w y\right) d y, w \in \mathbb{R}$, of the Airy function and performing a similar analysis around the stationary phase point $k_{0}=-1$ for $\xi=-v_{0}$ one obtains an asymptotic formula for the region not covered by (2.31)

$$
\begin{aligned}
S_{11}(j, t)= & \frac{1}{2 \beta \lambda_{0} t^{1 / 3}}\left[\mathrm{Ai}\left(\frac{j-v_{0} t}{\lambda_{0} t^{1 / 3}}\right)+\mathrm{Ai}\left(-\frac{j+v_{0} t}{\lambda_{0} t^{1 / 3}}\right)\right] \\
& +\mathcal{O}\left(t^{-1 / 2}\right), t \rightarrow \infty, \text { uniformly for }|j|<\left(v_{0}+\delta\right) t
\end{aligned}
$$

for $\delta>0$ (see e.g. [14]). Observe that due to the decay of $\operatorname{Ai}(w)$ for $w \rightarrow \pm \infty$, the Airy term is dominant roughly in the regions described by $v_{0} t-o(t)<|j|<v_{0} t+o\left((\ln t)^{2 / 3}\right)$.

From the arguments just presented it is not difficult to see that the derivation of (2.32) only uses the following properties of $f=f_{1}$ :

$$
f^{\prime \prime}(k)<0 \text { for all } 0<k \leq \frac{1}{2},
$$

together with

$$
f^{\prime \prime}(0)=0, \quad f^{\prime \prime \prime}(0)<0, \quad \text { and } \quad f(1-k)=f(k) \text { for all } 0 \leq k<\frac{1}{2} .
$$

Conditions (2.33) and (2.34) imply that statements (2.31) and (2.32) hold with $v_{0}=$ $\frac{f^{\prime}(0)}{2 \pi}>0$ and $\lambda_{0}=\frac{1}{2 \pi}\left|f^{\prime \prime \prime}(0) / 2\right|^{1 / 3}$.

It follows from equation (2.23) and from statement (a) of Lemma 2.5 below that the conditions of (2.34) are always satisfied in our model. Condition (2.33), however, might fail. Indeed, it is not hard to see that there exist open regions in the $\kappa$-space $\mathbb{R}_{+}^{m}$ where there always exist stationary phase points $k_{0} \in(0,1)$ of higher multiplicity, i.e. with $f^{\prime \prime}\left(k_{0}\right)=0$. In this situation the value of $v:=\frac{f^{\prime}\left(k_{0}\right)}{2 \pi}$ lies in the open interval $\left(-v_{0}, v_{0}\right)$ (cf. Lemma $2.5 \mathrm{~b}$ ). Then the decay rate of $S_{11}(j, t)$ for $j$ near $v t$ is at most of order $t^{-1 / 3}$. The decay is even slower (at least of order $t^{-1 / 4}$ ) if $f^{\prime \prime \prime}\left(k_{0}\right)=0$ holds in addition. We show in Theorem 2.7 that this may happen for $\boldsymbol{\kappa}$ in some submanifold of $\mathbb{R}_{+}^{m}$ of codimension 1 (see also Examples 2.8 and 2.9). Nevertheless, if $\kappa_{2}, \ldots, \kappa_{m}$ are sufficiently small in comparison to $\kappa_{1}$ then condition (2.33) is always satisfied as we show in Theorem 2.6(c).

Before stating our main results of this section, Theorems 2.6 and 2.7, we first summarize some more properties of the function $f$.

Lemma 2.5 Given $\left(\kappa_{1}, \ldots, \kappa_{m}\right)$ with $\kappa_{1}>0, \kappa_{m}>0$, and $\kappa_{j} \geq 0$ for $1<j<m$. Denote $f(k)=|\omega(k)|$ for $0 \leq k \leq 1$ as introduced in Lemma 2.3 and define $v_{0}:=\left(\sum_{s=1}^{m} s^{2} \kappa_{s}\right)^{\frac{1}{2}}$. Then the following holds:

(a) $f(0)=f^{\prime \prime}(0)=0, f^{\prime}(0)=2 \pi v_{0}$, and $f^{\prime \prime \prime}(0)=-\frac{2 \pi^{3}}{v_{0}} \sum_{s=1}^{m} s^{4} \kappa_{s}$.

(b) $f^{\prime}([0,1])=\left[-2 \pi v_{0}, 2 \pi v_{0}\right]$. $f^{\prime}$ attains its maximum only at $k=0$ and its minimum only at $k=1$.

(c) Fix $\kappa_{1}>0$. Then the map $f$ can be extended as a $C^{\infty}$-function of the variables $\left(k, \kappa_{2}, \ldots, \kappa_{m}\right)$ on the set $[0,1] \times[0, \infty)^{m-1}$.

Proof Statement (a) follows directly from the last formula in the proof of Lemma 2.3 and from the expansion $\operatorname{sinc}^{2}(x)=1-\frac{x^{2}}{3}+\mathcal{O}\left(x^{4}\right)$ for small values of $x$ :

$$
f(k)=2 \pi k\left(\sum_{s=1}^{m} s^{2} \kappa_{s} \operatorname{sinc}^{2}(\pi s k)\right)^{1 / 2}=2 \pi v_{0} k-\frac{\pi^{3}}{3 v_{0}}\left(\sum_{s=1}^{m} s^{4} \kappa_{s}\right) k^{3}+\mathcal{O}\left(k^{5}\right) .
$$


This representation also settles statement (c). As we know already $f^{\prime}(0)=2 \pi v_{0}=-f^{\prime}(1)$ we may establish statement (b) by verifying that $\left|f^{\prime}(k)\right|<2 \pi v_{0}$ holds for all $k \in(0,1)$. To this end we write $f=\left(\sum_{s=1}^{m} h_{s}^{2}\right)^{1 / 2}$ with $h_{s}(k)=2 \sqrt{\kappa_{s}} \sin (\pi s k)$. Using the CauchySchwarz inequality we obtain for $0<k<1$ that

$$
\begin{aligned}
\left|f^{\prime}(k)\right|= & \frac{\left|\sum_{s=1}^{m} h_{s}(k) h_{s}^{\prime}(k)\right|}{\left(\sum_{s=1}^{m} h_{s}^{2}(k)\right)^{1 / 2}} \leq\left(\sum_{s=1}^{m}\left(h_{s}^{\prime}\right)^{2}(k)\right)^{1 / 2} \\
& =2 \pi\left(\sum_{s=1}^{m} s^{2} \kappa_{s} \cos ^{2}(\pi s k)\right)^{1 / 2}<2 \pi v_{0},
\end{aligned}
$$

where the last inequality follows from $|\cos (\pi k)|<1$ and $\kappa_{1}>0$.

We are now ready to state our first main result in this section.

Theorem 2.6 Let $m \in \mathbb{N}$, fix $\delta>0$, denote $f(k)=|\omega(k)|$ as introduced in Lemma 2.3, and set

$$
v_{0}:=\sqrt{\sum_{s=1}^{m} s^{2} \kappa_{s}}, \quad \lambda_{0}:=\frac{1}{2}\left(\frac{1}{v_{0}} \sum_{s=1}^{m} s^{4} \kappa_{s}\right)^{1 / 3} .
$$

(a) For all $\alpha, \alpha^{\prime}=1,2,3$ we have rapid decay as $t \rightarrow \infty$, uniformly for $|j|>\left(v_{0}+\delta\right) t$, i.e.

$$
S_{\alpha \alpha^{\prime}}(j, t)=\mathcal{O}\left(t^{-\infty}\right) .
$$

(b) If $f^{\prime \prime}(k)<0$ for all $0<k \leq 1 / 2$ then as $t \rightarrow \infty$ the following holds uniformly for $|j|<\left(v_{0}+\delta\right) t$ :

$$
\begin{aligned}
& S_{11}(j, t)=\frac{1}{2 \beta \lambda_{0} t^{1 / 3}}\left[A i\left(\frac{j-v_{0} t}{\lambda_{0} t^{1 / 3}}\right)+A i\left(-\frac{j+v_{0} t}{\lambda_{0} t^{1 / 3}}\right)\right]+\mathcal{O}\left(t^{-1 / 2}\right)=S_{22}(j, t), \\
& S_{12}(j, t)=\frac{1}{2 \lambda_{0} t^{1 / 3} \beta}\left(A i\left(-\frac{j+v_{0} t}{\lambda_{0} t^{1 / 3}}\right)-A i\left(\frac{j-v_{0} t}{\lambda_{0} t^{1 / 3}}\right)\right)+\mathcal{O}\left(t^{-\frac{1}{2}}\right)=S_{21}(j, t), \\
& S_{33}(j, t)=\frac{1}{2 \beta^{2} \lambda_{0}^{2} t^{2 / 3}}\left[A i^{2}\left(\frac{j-v_{0} t}{\lambda_{0} t^{1 / 3}}\right)+A i^{2}\left(-\frac{j+v_{0} t}{\lambda_{0} t^{1 / 3}}\right)\right]+\mathcal{O}\left(t^{-5 / 6}\right)
\end{aligned}
$$

(c) For every $\kappa_{1}>0$ there exists $\varepsilon=\varepsilon\left(\kappa_{1}\right)>0$ such that for all $\left(\kappa_{2}, \ldots, \kappa_{m}\right) \in[0, \varepsilon)^{m-1}$ we have $f^{\prime \prime}(k)<0$ for all $0<k \leq 1 / 2$.

Proof The rapid decay claimed in statement (a) can be argued in the same way as (2.31) for $S_{11}=S_{22}$. Due to relations (2.18) and (2.28) one only needs to consider $S_{12}$ and $S_{21}$. Indeed, using Lemma 2.3 one may show that the imaginary parts of the integrands used in the representation of $S_{12}$ and $S_{21}$ in (2.39) below have smooth extensions to all $k \in \mathbb{R}$ that are 1-periodic. This is all that is needed because $\left|\frac{\partial}{\partial k} \phi_{ \pm}(k, j / t)\right|>2 \pi \delta$ by Lemma $\left.2.5 \mathrm{~b}\right)$ uniformly for $k \in[0,1 / 2]$ and $|j|>\left(v_{0}+\delta\right) t$.

We have already argued above that conditions (2.33), (2.34) suffice to derive the first claim of statement (b) with $v_{0}=\frac{f^{\prime}(0)}{2 \pi}>0$ and $\lambda_{0}=\frac{1}{2 \pi}\left|f^{\prime \prime \prime}(0) / 2\right|^{1 / 3}$. The expressions for $f^{\prime}(0)$ and $f^{\prime \prime \prime}(0)$ stated in Lemma 2.5 (a) justify the definitions of (2.35). 
Using the symmetry relations (2.23) and (2.24) we derive a representation for $S_{12}$ and $S_{21}$ that is suitable for a steepest descent analysis

$$
\begin{aligned}
S_{12}(j, t) & =\frac{1}{\beta} \int_{0}^{1 / 2}(\sin (f(k) t-2 \pi k j-\theta(k))+\sin (f(k) t+2 \pi k j+\theta(k))) \mathrm{d} k \\
& =\frac{1}{\beta} \Im \int_{0}^{1 / 2}\left(e^{i t \phi_{-}(k, j / t)} e^{-i \theta(k)}+e^{i t \phi_{+}(k, j / t)} e^{i \theta(k)}\right) d k \\
S_{21}(j, k) & =-\frac{1}{\beta} \Im \int_{0}^{1 / 2}\left(e^{i t \phi_{-}(k, j / t)} e^{i \theta(k)}+e^{i t \phi_{+}(k, j / t)} e^{-i \theta(k)}\right) d k
\end{aligned}
$$

where $\phi_{ \pm}(k, \xi)=f(k) \pm 2 \pi \xi k$ as in (2.29) above. Expanding for $k$ close to zero one obtains $\phi_{ \pm}(k, j / t)=2 \pi v_{0} k-\frac{1}{3}(2 \pi)^{3} \lambda_{0}^{3} k^{3} \pm 2 \pi k \frac{j}{t}+\mathcal{O}\left(k^{5}\right)$. Substituting $y=2 \pi \lambda_{0} t^{1 / 3} k$ leads to the asymptotic expression

$$
t \phi_{ \pm}(k, j / t)=\frac{v_{0} t \pm j}{\lambda_{0} t^{\frac{1}{3}}} y-\frac{1}{3} y^{3}+\mathcal{O}\left(t^{-\frac{2}{3}}\right) \quad \text { as } t \rightarrow \infty .
$$

Keeping in mind that $\theta(0)=\frac{\pi}{2}$ we obtain

$$
S_{12}(j, t)=\frac{1}{2 \lambda_{0} t^{1 / 3} \beta}\left(\operatorname{Ai}\left(-\frac{j+v_{0} t}{\lambda_{0} t^{1 / 3}}\right)-\operatorname{Ai}\left(\frac{j-v_{0} t}{\lambda_{0} t^{1 / 3}}\right)\right)+\mathcal{O}\left(t^{-\frac{1}{2}}\right)=S_{21}(j, t) .
$$

Regarding the expansion for $t \rightarrow \infty$ of $S_{33}(j, t)$ it follows immediately from the expression (2.28) and the expansions of $S_{\alpha \alpha^{\prime}}(j, t)$ with $\alpha, \alpha^{\prime}=1,2$.

Statement (c) follows from the continuous dependence of the derivatives $f^{\prime \prime}$ and $f^{\prime \prime \prime}$ on the parameters $\left(\kappa_{2}, \ldots, \kappa_{m}\right)$ (see Lemma $2.5 \mathrm{c}$ ) and from simple facts for the case of nearestneighbour interactions $f_{1}(k)=2 \sqrt{\kappa_{1}} \sin (\pi k)$ discussed above. Indeed, from $f^{\prime \prime}(0)=0$ and from $f_{1}^{\prime \prime \prime}(0)<0$ it follows that there exists such an $\varepsilon>0$ such that $f^{\prime \prime \prime}(k)<0$ and hence also $f^{\prime \prime}(k)<0$ for $k$ in some region $(0, \delta)$ uniformly in $\left(\kappa_{2}, \ldots, \kappa_{m}\right) \in[0, \varepsilon)^{m-1}$. As $f_{1}^{\prime \prime}(k)<-2 \pi^{2} \sqrt{\kappa_{1}} \sin (\pi \delta)$ for all $k \in[\delta, 1 / 2]$ we may prove the claim in this region by reducing the value of $\varepsilon$ if necessary.

Theorem 2.6 provides the leading order asymptotics of the limiting correlations $S_{\alpha \alpha^{\prime}}(j, t)$ for $t \rightarrow \infty$ in the simple situation that the second derivative of the dispersion relation is strictly negative on the open interval $(0,1)$ (cf. condition (2.33)). Moreover, statement (c) shows that there is a set of positive measure in parameter space $\kappa \in \mathbb{R}_{+}^{m}$ where this happens. For general values of $\boldsymbol{\kappa}$, however, different phenomena may appear. In particular, there might exist stationary phase points of higher order leading to slower time-decay of the correlations (see discussion before the statement of Lemma 2.5). By a naive count of variables and equations one might expect that decay rates $t^{-1 /(3+p)}$ occur on submanifolds of parameter space of dimension $m-p$. Theorem 2.7 shows that this is indeed the case for $p=1$. Moreover, we present in this situation a formula for the leading order contribution of the corresponding stationary phase points to the asymptotics of $S_{\alpha \alpha^{\prime}}(j, t)$. Despite being non-generic in parameter space it is interesting to note that decay rates $t^{-1 / 4}$ can be observed numerically (see Figs. 2 and 3). There is also a second issue that may arise if condition (2.33) fails. Namely, for $v \in\left(-v_{0}, v_{0}\right)$ there can be several values of $k \in\left(0, \frac{1}{2}\right]$ satisfying $f^{\prime}(k) \pm$ $2 \pi v=0$ so that the contributions from all these stationary points need to be added to describe the leading order behaviour for $j$ near $v t$. 
Theorem 2.7 Recall from (2.21) the formula for the dispersion relation

$$
f(k)=|\omega(k)|=\sqrt{2 \sum_{s=1}^{m} \kappa_{s}(1-\cos (2 \pi k s))} .
$$

(a) For $m \geq 3$ there is an $(m-1)$-parameter family of potentials for which there exists $k^{*}=k^{*}(\kappa) \in\left(0, \frac{1}{2}\right)$ with

$$
f^{\prime \prime}\left(k^{*}\right)=0, \quad f^{\prime \prime \prime}\left(k^{*}\right)=0, \quad f^{(i v)}\left(k^{*}\right) \neq 0, \quad \text { and } 0<v^{*}:=\frac{f^{\prime}\left(k^{*}\right)}{2 \pi}<v_{0},
$$

with $v_{0}$ as in (2.35). Set $\lambda^{*}:=\frac{1}{2 \pi}\left(\left|f^{(i v)}\left(k^{*}\right)\right| / 4 !\right)^{\frac{1}{4}}>0$. Then for $j \rightarrow \infty$ and $t \rightarrow \infty$ in such a way that

$$
\frac{v^{*} t-j}{\lambda^{*} t^{\frac{1}{4}}}
$$

is bounded, the contribution of the stationary phase point $k^{*}$ to the correlation functions is given by:

$$
\begin{aligned}
S_{11}(j, t), S_{22}(j, t) & : \frac{1}{2 \beta \pi \lambda^{*} t^{\frac{1}{4}}} \Re\left(e^{i t \phi_{-}\left(k^{*}, j / t\right)} \mathcal{P}_{ \pm}\left(\frac{v^{*} t-j}{\lambda^{*} t^{\frac{1}{4}}}\right)\right)+\mathcal{O}\left(t^{-\frac{1}{2}}\right), \\
S_{12}(j, t) & : \frac{1}{2 \beta \pi \lambda^{*} t^{\frac{1}{4}}} \Im\left(e^{i t \phi_{-}\left(k^{*}, j / t\right)-i \theta\left(k^{*}\right)} \mathcal{P}_{ \pm}\left(\frac{v^{*} t-j}{\lambda^{*} t^{\frac{1}{4}}}\right)\right)+\mathcal{O}\left(t^{-\frac{1}{2}}\right), \\
S_{21}(j, t) & :-\frac{1}{2 \beta \pi \lambda^{*} t^{\frac{1}{4}}} \Im\left(e^{i t \phi_{-}\left(k^{*}, j / t\right)+i \theta\left(k^{*}\right)} \mathcal{P}_{ \pm}\left(\frac{v^{*} t-j}{\lambda^{*} t^{\frac{1}{4}}}\right)\right)+\mathcal{O}\left(t^{-\frac{1}{2}}\right),
\end{aligned}
$$

where $\phi_{ \pm}(k, \xi)=f(k) \pm 2 \pi \xi k, \theta(k)=\arg \omega(k)$ as defined in Lemma 2.3, $\mathcal{P}_{ \pm}($a) denote the Pearcey integrals, $c f$. Appendix B:,

$$
\mathcal{P}_{ \pm}(a)=\int_{-\infty}^{\infty} e^{i\left( \pm y^{4}+a y\right)} d y, \quad a \in \mathbb{R}
$$

and $\mathcal{P}_{ \pm}$has to be chosen according to the sign of $f^{(i v)}\left(k^{*}\right)$. If $j \rightarrow-\infty$ with bounded $\left(v^{*} t+j\right) /\left(\lambda^{*} t^{1 / 4}\right)$ the contributions of the stationary point $k^{*}$ can be obtained from the ones presented in (2.41)-(2.43) by replacing $\phi_{-}$by $\phi_{+}, \theta$ by $-\theta$, and $j$ in the argument of $\mathcal{P}_{ \pm}$by $-j$.

(b) When $k^{*}=\frac{1}{2}$ one has $f^{\prime}(1 / 2)=0$ and $f^{\prime \prime \prime}(1 / 2)=0$ by the symmetry (2.23). For each $m \geq 2$ there is an $(m-1)$-parameter family of potentials so that $f^{\prime \prime}(1 / 2)=0$ and $f^{(i v)}(1 / 2) \neq 0$ holds in addition. In this case the contribution of the stationary phase point $k^{*}=1 / 2$ to the correlation functions in the asymptotic regime $t \rightarrow \infty$ with bounded $j / t^{\frac{1}{4}}$ is given by $\left(\lambda^{*}\right.$ defined as in statement (a) with $\left.k^{*}=\frac{1}{2}\right)$ 


$$
\begin{aligned}
S_{11}(j, t), S_{22}(j, t): & \frac{(-1)^{j}}{2 \beta \pi \lambda^{*} t^{\frac{1}{4}}} \Re\left(e^{i t f\left(\frac{1}{2}\right)} \mathcal{P}_{ \pm}\left(\frac{j}{\lambda^{*} t^{\frac{1}{4}}}\right)\right)+\mathcal{O}\left(t^{-\frac{1}{2}}\right) \\
S_{12}(j, t), S_{21}(j, t): & -\operatorname{sgn}\left(\sum_{\text {sodd }} \tau_{s}\right) \frac{(-1)^{j}}{2 \beta \pi \lambda^{*} t^{\frac{1}{4}}} \Im\left(e^{i t f\left(\frac{1}{2}\right)} \mathcal{P}_{ \pm}\left(\frac{j}{\lambda^{*} t^{\frac{1}{4}}}\right)\right)+\mathcal{O}\left(t^{-\frac{1}{2}}\right) \\
S_{33}(j, t): & \frac{1}{4 \beta^{2} \pi^{2}\left(\lambda^{*}\right)^{2} t^{\frac{1}{2}}}\left|\mathcal{P}_{ \pm}\left(\frac{j}{\lambda^{*} t^{\frac{1}{4}}}\right)\right|^{2}+\mathcal{O}\left(t^{-\frac{3}{4}}\right) .
\end{aligned}
$$

Proof We begin by proving formula (2.41) for the momentum or position correlations $S_{22}(j, t)=S_{11}(j, t)$ under the assumption that we have found a $k^{*} \in(0,1 / 2)$ for which all the relations of (2.40) are satisfied.

From (2.30) and Lemma 2.3 we obtain

$$
S_{11}(j, t)=S_{22}(j, t)=\frac{1}{\beta} \Re \int_{0}^{\frac{1}{2}}\left(e^{i t\left(f(k)+2 \pi k \frac{j}{t}\right)}+e^{i t\left(f(k)-2 \pi k \frac{j}{t}\right)}\right) d k .
$$

In order to compute the contribution of the stationary phase point $k^{*}$ to the large $t$ asymptotics of the integral in (2.46) we expand

$$
f(k)=f\left(k^{*}\right)+2 \pi v^{*}\left(k-k^{*}\right)+f^{(i v)}\left(k^{*}\right)\left(k-k^{*}\right)^{4} / 4 !+O\left(\left(k-k^{*}\right)^{5}\right) .
$$

Introducing the change of variables

$$
y=2 \pi \lambda^{*}\left(k-k^{*}\right) t^{\frac{1}{4}}, \quad \lambda^{*}=\frac{1}{2 \pi}\left(\left|f^{(i v)}\left(k^{*}\right)\right| / 4 !\right)^{\frac{1}{4}}
$$

one obtains

$$
t f(k)-2 \pi j k=t f\left(k^{*}\right)-2 \pi j k^{*}+y \frac{v^{*} t-j}{\lambda^{*} t^{\frac{1}{4}}} \pm y^{4}+\mathcal{O}\left(t^{-\frac{1}{4}}\right)
$$

where the \pm sign is determined by the sign of $f^{(i v)}\left(k^{*}\right)$. Then using the Pearcey integral (2.44), the expansion (2.41) can be derived in a straightforward way from (2.46). In a similar way the expansions (2.42) and (2.43) are obtained by applying the above analysis to the expression (2.39).

In the situation $k^{*}=1 / 2$ of statement (b) one uses in addition that $t \phi_{ \pm}(1 / 2, j / t)=$ $\operatorname{tf}(1 / 2) \pm j \pi, \omega(1 / 2)=-\sum_{s=1}^{m} \tau_{s}(1-\cos (\pi s))=-2 \sum_{s o d d} \tau_{s}$, see (2.21), and consequently $e^{ \pm i \theta(1 / 2)}=-\operatorname{sgn}\left(\sum_{s o d d} \tau_{s}\right)$. The leading order contribution of the stationary phase point $k^{*}=1 / 2$ to the integral representation of, say, $S_{12}$ in (2.39) is then given by

$$
-\operatorname{sgn}\left(\sum_{s \text { odd }} \tau_{s}\right) \frac{(-1)^{j}}{2 \beta \pi \lambda^{*} t^{\frac{1}{4}}} \Im\left(e^{i t f\left(\frac{1}{2}\right)}\left(\int_{-\infty}^{0} e^{i\left( \pm y^{4}-w y\right)} d y+\int_{-\infty}^{0} e^{i\left( \pm y^{4}+w y\right)} d y\right)\right)
$$

with $w=\frac{j}{\lambda^{*} t^{\frac{1}{4}}}$. In this way and with the help of (2.28) all relations of (2.45) can be deduced.

We now show the existence of a codimension 1 manifold in parameter space that exhibits such higher order stationary phase points in the situation of (b) where $k^{*}=1 / 2$. As we have $f^{\prime \prime \prime}(1 / 2)=0$ by symmetry (2.23) we only need to solve

$$
f^{\prime \prime}\left(\frac{1}{2}\right)=0 \text { which is equivalent to } \sum_{s=1}^{m} s^{2}(-1)^{s+1} \kappa_{s}=0 .
$$


The solution of the above equation is

$$
\kappa_{m}=\frac{(-1)^{m}}{m^{2}} \sum_{s=1}^{m-1} s^{2}(-1)^{s+1} \kappa_{s} .
$$

It is clear from the above relation that for $m$ even, choosing $\kappa_{1}$ sufficiently big one has $\kappa_{m}>0$ while for $m$ odd, it is sufficient to choose $\kappa_{s+1}>\frac{s^{2}}{(s+1)^{2}} \kappa_{s}>0, s$ odd and $1 \leq s \leq m-2$. Note that in the situation of (2.48) $f^{(i v)}\left(\frac{1}{2}\right) \neq 0$ holds iff $\sum_{s=1}^{m} \kappa_{s} s^{4}(-1)^{s+1} \neq 0$. This condition simply removes an $(m-2)$-dimensional plane from our manifold (2.48) which defines a hyperplane in the positive cone of the $m$-dimensional parameter space. Therefore we have found an $(m-1)$-parameter family of potentials such that the correlation functions decay as in (2.45).

Finally, we show for $m \geq 4$ our claim about the solution set of (2.40). The case $m=3$ is treated in Example 2.9. Our strategy is to first show that there exists a $\boldsymbol{\kappa}^{*}$ that satisfies $f^{\prime \prime}\left(1 / 4, \boldsymbol{\kappa}^{*}\right)=0, f^{\prime \prime \prime}\left(1 / 4, \boldsymbol{\kappa}^{*}\right)=0, f^{\prime}\left(1 / 4, \boldsymbol{\kappa}^{*}\right)>0$, and $f^{(i v)}\left(1 / 4, \boldsymbol{\kappa}^{*}\right) \neq 0$. We then invoke the Implicit Function Theorem to show the existence of the $(m-1)$-dimensional solution manifold, where the stationary phase point $k^{*} \sim 1 / 4$ may and will depend on the parameters. The conditions $f^{\prime \prime}\left(\frac{1}{4}, \kappa\right)=0$ and $f^{\prime \prime \prime}\left(\frac{1}{4}, \kappa\right)=0$ imply

$$
\begin{aligned}
f^{\prime \prime \prime}\left(\frac{1}{4}\right)=0 \rightarrow \sum_{\text {sodd }}(-1)^{\frac{s-1}{2}} s^{3} \kappa_{s}=0 \\
f^{\prime \prime}\left(\frac{1}{4}\right)=0 \rightarrow\left(2 \sum_{\text {sodd }} \kappa_{s}+2 \sum_{\text {seven }} \kappa_{s}\left(1-(-1)^{\frac{s}{2}}\right)\right) \sum_{\text {seven }} s^{2} \kappa_{s}(-1)^{\frac{s}{2}} \\
-\left(\sum_{\text {sodd }} s \kappa_{s}(-1)^{\frac{s-1}{2}}\right)^{2}=0 .
\end{aligned}
$$

One needs to treat the case $m$ odd and even separately. Here we consider only the case $m$ even. The odd case can be treated in a similar way. Equation (2.49) gives

$$
\kappa_{m-1}=\frac{(-1)^{\frac{m}{2}}}{(m-1)^{3}} \sum_{s o d d, s=1}^{m-3}(-1)^{\frac{s-1}{2}} s^{3} \kappa_{s} .
$$

If $m=2 \ell$ with $\ell$ even, a positive solution $\kappa_{m-1}$ exists, provided that $\kappa_{1}$ is sufficiently big. If $m=2 \ell$ with $\ell$ odd then one needs to require $0<\kappa_{s}<\frac{(s+2)^{3}}{s^{3}} \kappa_{s+2}$ for $s=1,5,9, \ldots, m-5$.

The equation (2.50) is a linear equation in $\kappa_{4}$ and we solve it for $\kappa_{4}$ obtaining

$$
\begin{gathered}
\kappa_{4}=\frac{1}{32} \frac{\left(\sum_{s \text { odd }, s=1}^{m-3} \kappa_{s}(-1)^{\frac{s-1}{2}} s\left(1-\frac{s^{2}}{(m-1)^{2}}\right)\right)^{2}}{\sum_{\text {sodd, }, s=1}^{m-3} \kappa_{S}\left(1+\frac{s^{3}(-1)^{\frac{m+s-1}{2}}}{(m-1)^{3}}\right)+\sum_{\text {seven }, s=2}^{m} \kappa_{s}\left(1-(-1)^{\frac{s}{2}}\right)} \\
+\frac{1}{16} \sum_{\text {seven }, s \neq 4, s=2}^{m} s^{2} \kappa_{s}(-1)^{\frac{s-2}{2}} .
\end{gathered}
$$

We observe that the first term in the above expression is always positive, while the second term is positive if we require that $\kappa_{s}>\frac{(s+2)^{2}}{s^{2}} \kappa_{s+2}>0$ for $s=6,10,14, \ldots, m-2$. The remaining two conditions $f^{\prime}(1 / 4)>0$ and $f^{(i v)}(1 / 4) \neq 0$ are easy to satisfy: the sign of 
$f^{\prime}(1 / 4)$ agrees with the sign of $\sum_{s o d d} s \kappa_{s}(-1)^{\frac{s-1}{2}}$ and can be made positive by choosing $\kappa_{1}$ sufficiently large. In the situation where (2.49) and (2.50) hold the fourth derivative $f^{(i v)}(1 / 4)$ does not vanish iff $\sum_{s \text { even }} s^{4} \kappa_{s}(-1)^{\frac{s}{2}} \neq 0$. This can be achieved by adjusting, for example, the value of $\kappa_{2}$. We have now shown that there exists $\kappa^{*} \in \mathbb{R}_{+}^{m}$ such that the first four derivatives of $f$ have all desired properties at $k=1 / 4$. In order to obtain the $(m-1)$ dimensional solution manifold in parameter space, we apply the Implicit Function Theorem to $F(k, \kappa):=\left(f^{\prime \prime}(k, \kappa), f^{\prime \prime \prime}(k, \kappa)\right)$. By a straight forward computation on sees that

$$
\operatorname{det}\left[\frac{\partial F}{\partial\left(k, \kappa_{4}\right)}\left(1 / 4, \kappa^{*}\right)\right]=-f^{(i v)}\left(1 / 4, \kappa^{*}\right) \frac{\partial f^{\prime \prime}}{\partial \kappa_{4}}\left(1 / 4, \kappa^{*}\right) \neq 0 .
$$

We can therefore solve $F(k, \kappa)=0$ near $\left(1 / 4, \boldsymbol{\kappa}^{*}\right)$ by choosing $\left(k, \kappa_{4}\right)$ as functions of the remaining parameters $\kappa_{j}$ with $j \neq 4$.

Example $2.8 m$ even. Choosing $\kappa_{s}=\frac{1}{s^{2}}$ for $s=1, \ldots, m$ one has that conditions (2.47) are satisfied and $f^{(i v)}\left(\frac{1}{2}\right)<0$.

For $\kappa_{s}=\frac{1}{s^{\alpha}}, s=1, \ldots, m-1,2<\alpha<3$, and $\kappa_{m}$ given by (2.48), there is $\alpha=\alpha(m)$ such that $\kappa_{m}<\kappa_{m-1}$.

$m$ odd. Choosing $\kappa_{s}=\frac{1}{s}$, for $s=1, \ldots m-1$, one has from $(2.48) \kappa_{m}=\frac{m-1}{2 m^{2}}<\kappa_{m-1}$ and $f^{(i v)}\left(\frac{1}{2}\right)>0$.

In all these examples the correlation functions $S_{\alpha \alpha^{\prime}}(j, t), \alpha, \alpha^{\prime}=1,2$ decrease as $t^{-\frac{1}{4}}$ near $j=0$.

Example 2.9 We consider the case $m=3$ and we want to get a potential that satisfies (2.40) with $v^{*}>0$. We chose as a critical point of $f(k)$ the point $k^{*}=\frac{1}{3}$ thus obtaining the equations

$$
\kappa_{2}=\frac{1}{8} \kappa_{1}, \quad \kappa_{3}=\frac{7}{72} \kappa_{1} .
$$

The speed of the peak is $v^{*}=\frac{\sqrt{2 \kappa_{1}}}{4}$ and $f^{(i v)}\left(\frac{1}{3}\right)=-\frac{68 \sqrt{6}}{6} \pi^{4} \sqrt{\kappa_{1}}$.

The correlation functions $S_{\alpha \alpha^{\prime}}(j, t), \alpha, \alpha^{\prime}=1,2$ decrease as $t^{-\frac{1}{4}}$ and $S_{33}(j, t)$ decreases like $t^{-\frac{1}{2}}$ as $t \rightarrow \infty$ and $j \sim v^{*} t$, see Fig. 3. Note that one may obtain a 2-parameter family of solutions of (2.40) by picking, for example, the particular solution related to $\kappa_{1}=1$ and by showing that the system of equations $\left(f^{\prime \prime}, f^{\prime \prime \prime}\right)(k, \kappa)=0$ can be solved near $(1 / 3,1,1 / 8$, $7 / 72$ ) by choosing $k$ and $\kappa_{3}$ as functions of $\kappa_{1}$ and $\kappa_{2}$ using the Implicit Function Theorem in the same way as at the end of the proof of Theorem 2.7.

\section{Complete Set of Integrals with Local Densities, Currents and potentials, and Some Numerics for Nonlinear Versions}

\subsection{Circulant Hierarchy of Integrals}

In this section we construct a complete set of conserved quantities that have local densities. The harmonic oscillator with short range interaction is clearly an integrable system. A set of integrals of motion is given by the harmonic oscillators in each of the Fourier variables: $\widehat{H}_{j}=$ $\frac{1}{2}\left(\left|\widehat{p}_{j}\right|^{2}+\left|\omega_{j}\right|^{2}\left|\widehat{q}_{j}\right|^{2}\right), j=0, \ldots \frac{N-1}{2}$. However, when written in the physical variables $\mathbf{p}$ and $\mathbf{q}$, the quantities 


$$
\widehat{H}_{j}=\frac{1}{2} \sum_{k, l=0}^{N-1} \mathcal{F}_{j, k} \overline{\mathcal{F}_{j, l}}\left(p_{k} p_{l}+\left|\omega_{j}\right|^{2} q_{k} q_{l}\right)
$$

depend on all components of the physical variables. We now construct integrals of motion each having a density that involves only a limited number of components of the physical variables and this number only depends on the range $m$ of interaction.

For this purpose we denote by $\left\{\mathbf{e}_{k}\right\}_{k=0}^{N-1}$ the canonical basis in $\mathbb{R}^{N}$.

Theorem 3.1 Let us consider the Hamiltonian

$$
H(\mathbf{p}, \mathbf{q})=\frac{1}{2} \mathbf{p}^{\top} \mathbf{p}+\frac{1}{2} \mathbf{q}^{\top} A \mathbf{q},
$$

with the symmetric circulant matrix $A$ as in (1.2), (1.3). Define the matrices $\left\{G_{k}\right\}_{k=1}^{M}$ to be the symmetric circulant matrix generated by the vector $\frac{1}{2}\left(\mathbf{e}_{k}+\mathbf{e}_{N-k}\right)$ and $\left\{S_{k}\right\}_{k=1}^{M}$ to be the antisymmetric circulant matrix generated by the vector $\frac{1}{2}\left(\mathbf{e}_{k}-\mathbf{e}_{N-k}\right)$. Then the family of Hamiltonians defined as

$$
\begin{aligned}
& H_{k}(\mathbf{p}, \mathbf{q})=\frac{1}{2} \mathbf{p}^{\boldsymbol{\top}} G_{k} \mathbf{p}+\frac{1}{2} \mathbf{q}^{\boldsymbol{\top}} T^{\boldsymbol{\top}} G_{k} T \mathbf{q}=\frac{1}{2} \sum_{j=0}^{N-1}\left[p_{j} p_{j+k}+r_{j} r_{j+k}\right], \\
& H_{k+\frac{N-1}{2}}(\mathbf{p}, \mathbf{q})=\mathbf{p}^{\top} T^{\top} S_{k} T \mathbf{q}=\frac{1}{2} \sum_{j=0}^{N-1}\left[\left(\sum_{\ell=0}^{m} \tau_{\ell} p_{j+\ell}\right)\left(r_{j+k}-r_{j-k}\right)\right],
\end{aligned}
$$

for $k=1, \ldots, \frac{N-1}{2}$ together with $H_{0}:=H$ forms a complete family $\left(H_{j}\right)_{0 \leq j \leq N-1}$ of integrals of motion that, moreover, is in involution.

Proof Observe first that the Hamiltonian $H_{0}=H$ is included in the description of formula (3.2) as $G_{0}$ equals the identity matrix. Using the symmetries $G_{k}^{\top}=G_{k}, 0 \leq k \leq(N-1) / 2$, the Poisson bracket $\{F, G\}=\left\langle\nabla_{\mathbf{q}} F, \nabla_{\mathbf{p}} G\right\rangle-\left\langle\nabla_{\mathbf{q}} G, \nabla_{\mathbf{p}} F\right\rangle$ may be evaluated in the form

$$
\begin{array}{ll}
\left\{H_{k}, H_{\ell}\right\}=\mathbf{q}^{\top}\left(T^{\top} G_{k} T G_{\ell}-T^{\top} G_{\ell} T G_{k}\right) \mathbf{p}, & \text { for } 0 \leq k, \ell \leq \frac{N-1}{2}, \\
\left\{H_{k}, H_{\ell}\right\}=\mathbf{p}^{\top}\left(T^{\top} S_{k} T T^{\top} S_{\ell} T-T^{\top} S_{\ell} T T^{\top} S_{k} T\right) \mathbf{q}, & \text { for } \frac{N+1}{2} \leq k, \ell \leq N-1, \\
\left\{H_{k}, H_{\ell}\right\}=\mathbf{q}^{\top} T^{\top} G_{k} T T^{\top} S_{\ell} T \mathbf{q}-\mathbf{p}^{\top} T^{\top} S_{\ell} T G_{k} \mathbf{p}, & \text { for } 0 \leq k \leq \frac{N-1}{2}, \frac{N+1}{2} \leq \ell \leq N-1 .
\end{array}
$$

All these expressions vanish. To see this, it suffices to observe that multiplication is commutative for circulant matrices and, for the bottom line, that $S_{\ell}$ is skew symmetric: $S_{\ell}^{\top}=-S_{\ell}$.

Now we introduce the local densities corresponding to the just defined integrals of motion

$$
e_{j}^{(k)}=\left\{\begin{array}{l}
\frac{1}{2}\left(p_{j} p_{j+k}+r_{j} r_{j+k}\right), \text { for } k=1, \ldots, \frac{N-1}{2} \\
\left(\sum_{l=0}^{m} \tau_{l} p_{j+l}\right)\left(r_{j+k}-r_{j-k}\right), \text { for } k=\frac{N+1}{2}, \ldots, N .
\end{array}\right.
$$

together with their correlation functions

$$
S_{(k+3, n+3)}^{(N)}(j, t):=\left\langle e_{j}^{(k)}(t) e_{0}^{(n)}(0)\right\rangle-\left\langle e_{j}^{(k)}(t)\right\rangle\left\langle e_{0}^{(n)}(0)\right\rangle .
$$

and limits

$$
S_{k, n}(j, t)=\lim _{N \rightarrow \infty} S_{k, n}^{(N)}(j, t) .
$$

We present explicit formulas for the limits $S_{k, n}$ in Appendix C: from which one can deduce that they have the same scaling behaviour as the energy-energy correlation function $S_{33}$ when $t \rightarrow \infty$. 


\subsection{Currents and Potentials}

In this subsection we write the evolution with respect to time of $r_{j}, p_{j}$ and $e_{j}$ in the form of a (discrete) conservation law by introducing the currents. Each conservation law has a potential function that is a Gaussian random variable. In the final part of this subsection we determine the leading order behaviour of the variance of this Gaussian random variable as $t \rightarrow \infty$ in the case of nearest neighbour interactions.

For introducing the currents we recall that $\mathbf{r}=T \mathbf{q}$ with $T$ as in (1.7). Then one has

$$
\begin{aligned}
& \dot{r}_{j}=\sum_{\ell} T_{j \ell} p_{\ell}=\sum_{\ell=1}^{m} \tau_{\ell}\left(p_{j+\ell}-p_{j}\right), \quad r_{j+N}=r_{j} \\
& \dot{p}_{j}=-\sum_{\ell} T_{\ell} r_{\ell}=\sum_{\ell=1}^{m} \tau_{\ell}\left(r_{j}-r_{j-\ell}\right), \quad p_{j+N}=p_{j}, j=0, \ldots, N-1 .
\end{aligned}
$$

To write the above equation in the form of a discrete conservation law we introduce the local currents

$$
\mathcal{J}_{j}^{(r)}:=\sum_{s=0}^{m-1} p_{j+1+s} \sum_{\ell=s+1}^{m} \tau_{\ell}, \quad \mathcal{J}_{j}^{(p)}:=\sum_{s=1}^{m} r_{j+1-s} \sum_{\ell=s}^{m} \tau_{\ell} .
$$

Then the equations of motion (3.6) can be written in the form

$$
\begin{aligned}
& \dot{r}_{j}=\mathcal{J}_{j}^{(r)}-\mathcal{J}_{j-1}^{(r)} \\
& \dot{p}_{j}=\mathcal{J}_{j}^{(p)}-\mathcal{J}_{j-1}^{(p)}, \quad j=0, \ldots, N-1 .
\end{aligned}
$$

From the above equations it is clear that the momentum $p_{j}$ and the generalized elongation $r_{j}$ are locally conserved. The evolution of the energy $e_{j}:=\frac{1}{2} p_{j}^{2}+\frac{1}{2} r_{j}^{2}$ at position $j$ takes the form

$$
\dot{e}_{j}=\mathcal{J}_{j}^{(e)}-\mathcal{J}_{j-1}^{(e)}, \quad \mathcal{J}_{j}^{(e)}=\sum_{s=1}^{m} \tau_{s} \sum_{\ell=0}^{s-1} r_{j+1-s+\ell} p_{j+1+\ell} .
$$

We remark that all the currents $\mathcal{J}_{j}^{(r)}, \mathcal{J}_{j}^{(p)}$ and $\mathcal{J}_{j}^{(e)}$ are local quantities in the variables $\mathbf{q}$ and p. Observe furthermore that the total current $\sum_{j} \mathcal{J}_{j}^{(p)}$ for the momentum is a multiple of the total stretch and that the total current $\sum_{j} \mathcal{J}_{j}^{(r)}$ for the stretch is a multiple of the total momentum. Therefore these two total currents are conserved quantities. However, the total current for the energy $\sum_{j} \mathcal{J}_{j}^{(e)}$ is not a conserved quantity. Of course, one could easily remedy this by simply subtracting the mean total current from each $\mathcal{J}_{j}^{(e)}$ at the cost of losing the local nature of the current. It is an interesting question whether one can find more integrals of motion (besides momentum and stretch) with corresponding currents that are both local quantities in the variables $\mathbf{q}$ and $\mathbf{p}$ so that the currents are conserved. To the best of our knowledge this is an open question even in the simplest case of linear nearest-neighbour interactions.

We recall the notation of the introduction

$$
\boldsymbol{u}(j, t)=\left(r_{j}(t), p_{j}(t), e_{j}(t)\right),
$$


and we introduce the vector of currents $\boldsymbol{J}(j, t)=\left(\mathcal{J}_{j}^{(r)}(t), \mathcal{J}_{j}^{(p)}(t), \mathcal{J}_{j}^{(e)}(t)\right)$. The equations of motion take the compact form

$$
\frac{d}{d t} \boldsymbol{u}(j, t)=\boldsymbol{J}(j, t)-\boldsymbol{J}(j-1, t) .
$$

We define a potential function for the above conservation law

$$
\boldsymbol{\Phi}(j, t):=\int_{0}^{t} \boldsymbol{J}\left(j, t^{\prime}\right) d t^{\prime}+\sum_{\ell=0}^{j} \mathbf{u}(\ell, 0)
$$

Then it is straightforward to verify that $\boldsymbol{\Phi}_{t}(j, t)=\boldsymbol{J}(j, t)$ and $\boldsymbol{\Phi}(j, t)-\boldsymbol{\Phi}(j-1, t)=\mathbf{u}(j, t)$. The quantities $\Phi_{1}(j, t)$ and $\Phi_{2}(j, t)$ can be expressed as sums of independent centered Gaussian random variables and are therefore also Gaussian random variables with zero mean and variance $\left\langle\left(\Phi_{1}(j, t)\right)^{2}\right\rangle$ and $\left\langle\left(\Phi_{2}(j, t)\right)^{2}\right\rangle$, where all the averages are taken with respect to the distribution (1.9), see also (2.10). We calculate the variance for the case of the harmonic oscillator with nearest neighbour interactions. In this particular case

$$
\begin{aligned}
& \Phi_{1}(j, t)=\sqrt{\kappa_{1}} \int_{0}^{t} p_{j+1}\left(t^{\prime}\right) d t^{\prime}+\sum_{\ell=0}^{j} r_{\ell}(0)=\sqrt{\kappa_{1}}\left(q_{j+1}(t)-q_{0}(0)\right) \\
& \Phi_{2}(j, t)=\sqrt{\kappa_{1}} \int_{0}^{t} r_{j}\left(t^{\prime}\right) d t^{\prime}+\sum_{\ell=0}^{j} p_{\ell}(0) .
\end{aligned}
$$

After some lengthy calculations one obtains:

$$
\begin{aligned}
& \lim _{N \rightarrow \infty}\left\langle\left(\Phi_{1}(j, t)\right)^{2}\right\rangle=\frac{2 \kappa_{1}}{\beta} \int_{0}^{1}|\omega(k)|^{-2}[1-\cos (|\omega(k)| t) \cos (2 \pi(j+1) k)] d k \\
& \lim _{N \rightarrow \infty}\left\langle\left(\Phi_{2}(j, t)\right)^{2}\right\rangle=\frac{2 \kappa_{1}}{\beta} \int_{0}^{1}|\omega(k)|^{-2}(1-\cos (|\omega(k)| t)) \cos (2 \pi(j+1) k) d k+\frac{j+1}{\beta} .
\end{aligned}
$$

Evaluating the r.h.s. of the above expressions in the limit $t \rightarrow \infty$ we arrive to the following theorem.

Theorem 3.2 In the limit $N \rightarrow \infty$ and $t \rightarrow \infty$ the quantities $\Phi_{1}(j, t)$ and $\Phi_{2}(j, t)$ defined in (3.11) are Gaussian random variables that have the following large t behaviour:

$$
\lim _{N \rightarrow \infty} \Phi_{1}(j, t)=\mathcal{N}\left(0, \sigma_{1}^{2}\right) \quad \text { and } \quad \lim _{N \rightarrow \infty} \Phi_{2}(j, t)=\mathcal{N}\left(0, \sigma_{2}^{2}\right) .
$$

The leading order behaviour of the variances $\sigma_{1}^{2}$ and $\sigma_{2}^{2}$ agrees. In the physically interesting region $\frac{|j|}{t} \leq \sqrt{\kappa_{1}}$ it is given by

$$
\sigma_{1}^{2}=\frac{t \sqrt{\kappa_{1}}}{\beta}+\mathcal{O}\left(t^{\frac{1}{3}}\right)=\sigma_{2}^{2} .
$$

The proof of the above theorem relies on steepest descent analysis of the oscillatory integrals in (3.13). But because the integrand is actually quite large $\left(\sim C t^{2}\right)$ near $k=0$, we consider the following Cauchy-type integral instead,

$$
F_{0}(z)=\frac{1}{2 \pi^{2} \beta} \int_{-1 / 2}^{1 / 2} \frac{1-\cos (|\omega(k)| t)}{(k-z)^{2}} \cos (2 \pi(j+1) k) d k,
$$


which gives the leading order asymptotic behaviour of the integrals appearing in (3.13), since

$$
\frac{2 \kappa_{1}}{\beta} \int_{-1 / 2}^{1 / 2}|\omega(k)|^{-2}(1-\cos (|\omega(k)| t)) \cos (2 \pi(j+1) k) d k-F_{0}(0) \rightarrow 0 \text { as } t, j \rightarrow \infty .
$$

For $\frac{|j|}{t}<(1-\epsilon) \sqrt{\kappa_{1}}, \epsilon>0$, the analysis of $F_{0}(z)$ is quite straightforward - a standard stationary phase calculation combined with a contour deformation to permit the evaluation at $z=0$. For $t$ and $j$ growing to $\infty$ such that $\frac{|j|}{t} \approx \sqrt{\kappa_{1}}$, the analysis is more complicated because the point of stationary phase is encroaching upon the origin, where the integrand itself is actually large as $t \rightarrow \infty$. For this case, one must construct a local parametrix, following quite closely the analysis presented in [5], and we omit the details of this analysis. In order to analyse $\Phi_{1}$ observe that the difference of the integrals in relations (3.12) and (3.13) is given by $\int_{0}^{1}|\omega(k)|^{-2}[1-\cos (2 \pi(j+1) k)] d k$ which can also be treated by a stationary phase calculation combined with a contour deformation.

\subsection{Nonlinear Regime}

In this section we consider a nonlinear perturbation of the harmonic oscillators with short range interactions of the form

$$
\begin{aligned}
H(\mathbf{p}, \mathbf{q})= & \sum_{j=0}^{N-1} \frac{p_{j}^{2}}{2}+\sum_{s=1}^{m} \kappa_{s}\left(\frac{1}{2} \sum_{j=0}^{N-1}\left(q_{j}-q_{j+s}\right)^{2}\right. \\
& \left.+\frac{\chi}{3} \sum_{j=0}^{N-1}\left(q_{j}-q_{j+s}\right)^{3}+\frac{\gamma}{4} \sum_{j=0}^{N-1}\left(q_{j}-q_{j+s}\right)^{4}\right) .
\end{aligned}
$$

We consider Examples 2.8 and 2.9 with different strengths of nonlinearity namely

$$
\begin{aligned}
& m=2, \kappa_{1}=1, \kappa_{2}=\frac{1}{4},\left\{\begin{array}{l}
\chi=0.01 \text { and } \gamma=0.001 \\
\chi=0.1 \text { and } \gamma=0.01
\end{array}\right. \\
& m=3, \kappa_{1}=1, \kappa_{2}=\frac{1}{8}, \kappa_{2}=\frac{7}{72},\left\{\begin{array}{l}
\chi=0.01 \text { and } \gamma=0.001 \\
\chi=0.1 \text { and } \gamma=0.01
\end{array} .\right.
\end{aligned}
$$

We numerically compute and study the correlatios functions for these systems sampling the initial conditions according to the Gibbs measures of just their harmonic part at temperature $\beta^{-1}=1$.

In the weakly nonlinear case, the fastest peaks of the correlation functions scale numerically according to the Airy parametrices (cf. Theorem 2.6) as can be deduced from the top pictures in Figs. 4, 5 while for stronger nonlinearity the fastest peaks seem to scale like $t^{\frac{2}{3}}$ in equation (1.11), see bottom figures in Figs. 4, 5. The non generic peaks that are present in the linear cases and scale like $t^{1 / 4}$ have a fast decay in the case of strong nonlinearity. However for weak nonlinearities, the central peak in the top left Fig. 4, still scales in time like $t^{-\frac{1}{4}}$. Indeed performing a regression analysis of the log-log plot one can see a scaling like $t^{-0.267}$ that is slightly faster then $t^{-\frac{1}{4}}$ (see Fig. 6).

Acknowledgements This manuscript was initiated during the research in pairs that took place in May 2019 at the Centre International des Rescontres mathématiques (CIRM), Luminy, France during the chair Morlet 
semester "Integrability and randomness in mathematical physics". The authors thank CIRM for the generous support, excellent work environment, and kind hospitality. K.M. was supported in part by the National Science Foundation under grant DMS-1733967. T.G. and G.M. acknowledge support from the European Union's H2020 research and innovation program under the Marie Skłowdoska-Curie Grant No. 778010 IPaDEGAN. We thank Manuela Girotti for related initial calculations in the case of nearest-neighbour interactions and the unknown referee for posing the question of the conservation of total currents. We also thank Giuseppe Pitton for sharing his codes with us, and for useful discussions.

Funding Open Access funding enabled and organized by Projekt DEAL.

Open Access This article is licensed under a Creative Commons Attribution 4.0 International License, which permits use, sharing, adaptation, distribution and reproduction in any medium or format, as long as you give appropriate credit to the original author(s) and the source, provide a link to the Creative Commons licence, and indicate if changes were made. The images or other third party material in this article are included in the article's Creative Commons licence, unless indicated otherwise in a credit line to the material. If material is not included in the article's Creative Commons licence and your intended use is not permitted by statutory regulation or exceeds the permitted use, you will need to obtain permission directly from the copyright holder. To view a copy of this licence, visit http://creativecommons.org/licenses/by/4.0/.

\section{Appendices}

\section{A: Proof of Proposition 2.2}

Proof In view of the notation introduced in (1.3), (1.4), and (1.7) the proof of Proposition 2.2 amounts to showing the existence of $\tau_{0}, \ldots, \tau_{m} \in \mathbb{R}$ satisfying $\sum_{s=0}^{m} \tau_{s}=0$ such that

$$
\begin{aligned}
Q\left(z^{-1}\right) Q(z) & =\ell(z) \quad \text { for all } z \in \mathbb{C} \backslash\{0\}, \text { where } \\
Q(z) & =\tau_{0}+\tau_{1} z+\ldots+\tau_{m} z^{m} \text { and } \\
\ell(z) & =-\kappa_{m} z^{-m}-\ldots-\kappa_{1} z^{-1}+a_{0}-\kappa_{1} z-\ldots-\kappa_{m} z^{m} .
\end{aligned}
$$

The existence of the $\tau_{j}$ 's is a consequence of the Fejér-Riesz lemma. For the convenience of the reader we present a proof following the presentation in [16, pg. $117 \mathrm{f}]$. Denote by $P$ the polynomial of degree $2 m$ given by $P(z):=z^{m} \ell(z)$. Observe that for all $x \in \mathbb{R}$ we have

$$
\ell\left(e^{i x}\right)=a_{0}-2 \sum_{j=1}^{m} \kappa_{j} \cos (j x) \geq a_{0}-2 \sum_{j=1}^{m} \kappa_{j}=0 .
$$

By the positivity of $\kappa_{1}$ equality holds in the inequality above iff $\cos (x)=1$. This implies that $P$ has no zeros on the unit circle $|z|=1$ except for $z=1$. We denote by $\eta_{k}, 1 \leq k \leq r_{<}$, the zeros of $P$ that lie within the unit disc $\left|\eta_{k}\right|<1$ and by $\xi_{k}, 1 \leq k \leq r_{>}$, the zeros of $P$ with $\left|\xi_{k}\right|>1$, recorded repeatedly according to their multiplicities, so that

$$
P(z)=-\kappa_{m}(z-1)^{r_{0}} \prod_{k=1}^{r_{<}}\left(z-\eta_{k}\right) \prod_{k=1}^{r_{>}}\left(z-\xi_{k}\right) .
$$

Using the uniqueness of such a factorization for any polynomial together with the relation $z^{2 m} P\left(z^{-1}\right)=P(z)$ one obtains that $r_{<}=r_{>}$and that the zeros can be listed in such a way that $\eta_{k}=\xi_{k}^{-1}$ for all $1 \leq k \leq r_{<}$. Moreover, we learn that $r_{0}$ is even with $1 \leq \varrho:=r_{0} / 2=m-r_{<}$. Now it follows from formula (A.2) that 


$$
\begin{aligned}
l(z) & =z^{-m} P(z) \\
& =c\left(z^{-1}-1\right)^{\varrho}(z-1)^{\varrho} \prod_{k=1}^{r_{<}}\left(z^{-1}-\xi_{k}\right) \prod_{k=1}^{r_{<}}\left(z-\xi_{k}\right) \\
\text { with } c: & :=-\kappa_{m}(-1)^{\varrho} \prod_{k=1}^{r_{<}}\left(-\xi_{k}^{-1}\right) \neq 0 .
\end{aligned}
$$

Choosing $d \in \mathbb{C}$ with $d^{2}=c$ we see that $Q(z):=d(z-1)^{\varrho} \prod_{k=1}^{r_{<}}\left(z-\xi_{k}\right)$ satisfies (A.1). Next we show that the coefficients of the polynomial $Q$ are real. To this end observe that $P$ has real coefficients and therefore all non-real zeros of $P$ come in complex conjugate pairs with equal multiplicities. Therefore the polynomial $d^{-1} Q(z)=\sum_{j=0}^{m} s_{j} z^{j}$ has only real coefficients $s_{j}$. Relation (A.1) implies $a_{0}=d^{2} \sum_{j=0}^{m} s_{j}^{2}$. Consequently, $d^{2}$ is the quotient of two positive numbers and $d$ must be real. Thus we have $\tau_{j}=d s_{j} \in \mathbb{R}$ for all $0 \leq j \leq m$. We complete the proof by arguing that $\sum_{s=0}^{m} \tau_{s}=0$ and $\left(\sum_{s=1}^{m} s \tau_{s}\right)^{2}=\sum_{s=1}^{m} s^{2} \kappa_{s}$ hold true. This can be deduced from (A.1) via $Q(1)^{2}=\ell(1)=0$ and $-2 Q^{\prime}(1)^{2}=\ell^{\prime \prime}(1)=-\sum_{s=1}^{m} 2 s^{2} \kappa_{s}$.

\section{B: Pearcey Integral}

The general Pearcey integral is defined as

$$
\bar{P}(b, a):=\int_{-\infty}^{\infty} e^{i\left(t^{4}+b t^{2}+a t\right)} d t, \quad 0 \leq \arg b \leq \pi, \quad a \in \mathbb{R} .
$$

This integral describes cusp singularities in physical phenomena, like the semiclassical limit of the linear Schrödinger equation. The integral (B.1), after a rotation of the integration path through an angle of $\pi / 8$ that removes the rapidly oscillatory term $e^{i t^{4}}$, can be written in the form $\bar{P}(b, a)=2 e^{i \pi / 8} P\left(b e^{-i \pi / 4}, a e^{i \pi / 8}\right)$, with

$$
P(b, a):=\int_{0}^{\infty} e^{-t^{4}-b t^{2}} \cos (a t) d t
$$

We are interested in the case $b=0$. The corresponding integral is absolutely convergent for all complex values of $a$ and represents the analytic continuation of the Pearcey integral. For the Pearcey integral $\mathcal{P}_{+}(a)$ defined in (2.44) we obtain

$$
\mathcal{P}_{+}(a)=2 e^{i \pi / 8} P\left(0, a e^{i \pi / 8}\right)
$$

Note that the integral $\mathcal{P}_{-}(a)$, also defined in (2.44), can be related to the function $P$ by rotating the integration path by an angle of $-\pi / 8$. This gives $\mathcal{P}_{-}(a)=2 e^{-i \pi / 8} P\left(0, a e^{-i \pi / 8}\right)$. From this we learn that $\mathcal{P}_{-}(\bar{a})=\overline{\mathcal{P}_{+}(a)}$. On the reals we therefore have

$$
\mathcal{P}_{-}(a)=\overline{\mathcal{P}_{+}(a)}, \quad a \in \mathbb{R}
$$




\section{C: Integrals of Motion Correlation Functions}

Here, for completeness, we report the limiting correlation functions for the integral of motions as defined in (3.5). Using the notation $f(k)=|\omega(k)|$ introduced in Lemma 2.3 they are:

$$
\begin{aligned}
& S_{k+3, n+3}(j, t)=\frac{1}{2 \beta^{2}} \int_{0}^{1} \int_{0}^{1} \cos (f(x) t) \cos (f(y) t) \cos (2 \pi x(j-n)) \cos (2 \pi y(j+k)) \\
& \quad+\cos (f(x) t) \cos (f(y) t) \cos (2 \pi x j) \cos (2 \pi y(j+k-n)) \\
& \quad+\sin (f(x) t) \sin (f(y) t) \cos (2 \pi x(j-n)) \cos (2 \pi y(j+k)) \cos (\theta(x)) \cos (\theta(y)) \\
& \quad+\sin (f(x) t) \sin (f(y) t) \sin (2 \pi x(j-n)) \sin (2 \pi y(j+k)) \sin (\theta(x)) \sin (\theta(y)) \mathrm{d} x \mathrm{~d} y
\end{aligned}
$$

for $k, n \leq \frac{N-1}{2}$,

$$
\begin{aligned}
& S_{n+3, k+3}(j, t) \\
& =\frac{1}{2 \beta^{2}} \int_{0}^{1} \int_{0}^{1} f(x) f(y) \sin (f(x) t) \sin (f(y) t) \sin (2 \pi x j) \sin (2 \pi y j) \sin (2 \pi x n) \sin (2 \pi y k) \\
& \quad+f^{2}(x) \cos (f(x) t) \cos (f(y) t) \cos (2 \pi x j) \cos (2 \pi y j) \sin (2 \pi y n) \sin (2 \pi y k) \mathrm{d} x \mathrm{~d} y
\end{aligned}
$$

for $k, n>\frac{N-1}{2}$ and

$$
\begin{aligned}
& S_{n+3, k+3}(j, t) \\
& =\frac{1}{2 \beta^{2}} \int_{0}^{1} \int_{0}^{1} \cos (2 \pi x j-\theta(x)) \cos (2 \pi y j) \sin (2 \pi y k) \sin (2 \pi y n) \sin ((f(x)+f(y)) t) \\
& \quad+\cos (2 \pi x j-\theta(x)) \sin (2 \pi y j) \sin (2 \pi y k) \cos (2 \pi y n) \sin ((f(x)-f(y)) t) \mathrm{d} x \mathrm{~d} y
\end{aligned}
$$

for $k>\frac{N-1}{2}, n \leq \frac{N-1}{2}$.

\section{D: Numerical Computation}

The numerical computations have been implemented with Python software, all codes are available on GitHub [11]. Figures 1 and 3 are the result of the numerical evaluation via the standard routine numpy . trapz of the integrals in (2.25)-(2.28) for various values of $j$ and $t$ and then we just added the Airy function (1.16) and the Pearcey integral (2.45).

To obtain Fig. 4 we proceed in the following way. First we sampled a random initial data according to the Gibbs measure defined by the corresponding harmonic part of (3.18), namely the Hamiltonian of Example 2.8 with $m=2$. We let these data evolve according to the Hamilton equations (3.18) and compute the values of the correlations function. Then we repeated this procedure $4 \times 10^{6}$ times and we averaged the values of the correlations functions. On the left panel we plot the correlation functions, instead on the right one we focus on the extreme peak and we guess a proper scaling depending on the size of the perturbation. Figure 5 is made in a similar way. In this case the nonlinear potential has the same harmonic part as Example 2.9.

In Fig. 6 we focus our attention on the central peak of the chain with potential as is Fig. 4. We follow the same procedure as before and plot in logarithmic scale the average scaling of the highest peak in the center of the chain. We decide to plot the average height of this peak since it is highly oscillatory and it is difficult to precisely track the oscillations. 


\section{References}

1. Corwin, I.: The Kardar-Parisi-Zhang equation and universality class. In: Random Matrices: Theory and Applications. World Scientific, Singapore (2012)

2. Dematteis, G., Rondoni, L.P.D., De Vita, F., Onorato, M.: Coexistence of ballistic and Fourier regimes in the beta-FPUT lattice. Phys. Rev. Lett. 125, 024101 (2020)

3. Dhar, A.: Heat transport in low-dimensional systems. Adv. Phys. 57, 457-537 (2008)

4. Kriecherbauer, T., Krug, J.: A pedestrian's view on interacting particle systems, KPZ universality and random matrices. J. Phys. A 43, 403001403001 (2010)

5. Kriecherbauer, T., Kuijlaars, A.B.J., McLaughlin, K.D.T.-R., Miller, P.D.: Locating the zeros of partial sums of $e^{z}$ with Riemann-Hilbert methods. In: Aswi, A. (ed.) Integrable Systems and Random Matrices. Contemporary Mathematics, vol. 458, pp. 183-195. American Mathematical Society, Providence, RI (2008)

6. Kundu, A., Dhar, A.: Equilibrium dynamical correlations in the toda chain and other integrable models. Phys. Rev. E 94, 062130 (2016)

7. Lepri, S. (ed.): Thermal Transport in Low Dimensions: From Statistical Physics to Nanoscale Heat Transfer. Lecture Notes in Physics, vol. 921. Springer, New York (2016)

8. Lepri, S., Livi, R., Politi, A.: Heat Transport in Low Dimensions: Introduction and Phenomenology, in Thermal Transport in Low Dimensions. Lecture Notes in Physics, vol. 921, pp. 1-37. Springer, Cham (2016)

9. Lukkarinen, J.: Kinetic theory of phonons in weakly anharmonic particle chains. In: Thermal transport in low dimensions. Lecture Notes in Physics, vol. 921, pp. 159-214. Springer, Cham (2016)

10. Mazur, P., Montroll, E.: Poincaré cycles, ergodicity, and irreversibility in assemblies of coupled harmonic oscillators. J. Math. Phys. 1, 70-84 (1960)

11. Mazzuca, G.: Correlation functions for the short range FPUT chain (2020). https://github.com/gmazzuca/ CorrelationFunctionShortRangeFPUT

12. Mendl, C.B., Spohn, H.: Low temperature dynamics of the one-dimensional discrete nonlinear Schrödinger equation. J. Stat. Mech. Theory Exp. 35, P08028 (2015)

13. Mendl, C.B., Spohn, H.: Shocks, rarefaction waves, and current fluctuations for anharmonic chains. J. Stat. Phys. 166, 841-875 (2017)

14. Miller, P.D.: Applied Asymptotic Analysis. Graduate Studies in Mathematics, American Mathematical Society, Providence, RI (2006)

15. Rieder, Z., Lebowitz, J.L.: Properties of a harmonic crystal in a stationary nonequilibrium state. J. Math. Phys. 8, 1073 (1967)

16. Riesz, F., Sz.-Nagy, B.: Functional analysis, Dover Books on Advanced Mathematics. Dover, New York. Translated from the second French edition by Leo F. Boron, Reprint of the 1955 original (1990)

17. Spohn, H.: Fluctuating hydrodynamics approach to equilibrium time correlations for anharmonic chains. In: Thermal transport in low dimensions. Lecture Notes in Physics, vol. 921, pp. 107-158. Springer, Cham (2016)

18. Spohn, H.: Nonlinear fluctuating hydrodynamics for anharmonic chains. J. Stat. Phys. 154, 1191-1227 (2014)

19. Spohn, H.: Ballistic space-time correlators of the classical Toda lattice. J. Phys. A 53, 265004 (2020)

20. Tracy, C.A., Widom, H.: Level-spacing distributions and the Airy kernel. Commun. Math. Phys. 159, 151-174 (1994)

Publisher's Note Springer Nature remains neutral with regard to jurisdictional claims in published maps and institutional affiliations. 